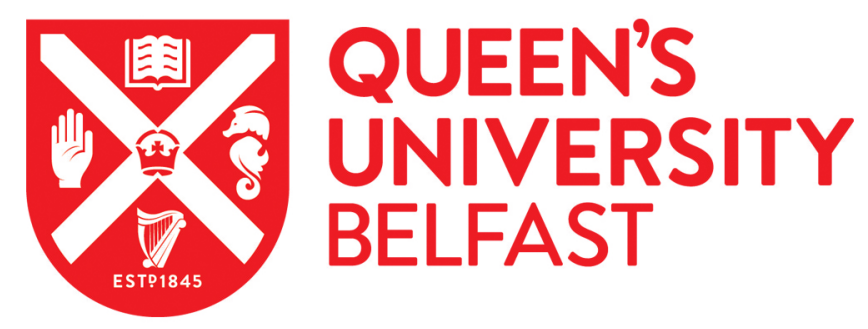

\title{
Glaucoma in the Northern Ireland Cohort for the Longitudinal Study of Ageing (NICOLA): Cohort profile, prevalence, awareness and associations
}

McCann, P., Hogg, R., Wright, D. M., Pose-Bazarra, S., Chakravarthy, U., Peto, T., Cruise, S., McGuinness, B., Young, I. S., Kee, F., \& Azuara-Blanco, A. (2020). Glaucoma in the Northern Ireland Cohort for the Longitudinal Study of Ageing (NICOLA): Cohort profile, prevalence, awareness and associations. British Journal of Ophthalmology, [315330]. https://doi.org/10.1136/bjophthalmol-2019-315330

Published in:

British Journal of Ophthalmology

Document Version:

Peer reviewed version

Queen's University Belfast - Research Portal:

Link to publication record in Queen's University Belfast Research Portal

\section{Publisher rights}

Copyright $2020 \mathrm{BMJ}$. This work is made available online in accordance with the publisher's policies. Please refer to any applicable terms of use of the publisher.

\section{General rights}

Copyright for the publications made accessible via the Queen's University Belfast Research Portal is retained by the author(s) and / or other copyright owners and it is a condition of accessing these publications that users recognise and abide by the legal requirements associated with these rights.

Take down policy

The Research Portal is Queen's institutional repository that provides access to Queen's research output. Every effort has been made to ensure that content in the Research Portal does not infringe any person's rights, or applicable UK laws. If you discover content in the

Research Portal that you believe breaches copyright or violates any law, please contact openaccess@qub.ac.uk. 


\section{British Journal of Ophthalmology}

\section{Glaucoma in the Northern Ireland Cohort for the Longitudinal Study of Ageing (NICOLA): cohort profile, prevalence, awareness and associations}

\begin{tabular}{|r|l|}
\hline Journal: & British Journal of Ophthalmology \\
\hline Manuscript ID & bjophthalmol-2019-315330.R1 \\
\hline Article Type: & Clinical science \\
\hline Author: & 13-Dec-2019 \\
\hline Complete List of Authors: & $\begin{array}{l}\text { McCann, Paul; Queen's University Belfast, Centre for Public Health } \\
\text { Hogg, Ruth; Queen's University Belfast School of Medicine Dentistry and } \\
\text { Biomedical Sciences, Centre for Public Health } \\
\text { Wright, David; Queen's University Belfast, Centre for Public Health } \\
\text { Pose-Bazarra, Sara; University Hospital Complex of Ferrol } \\
\text { Chakravarthy, Usha; Queens University, } \\
\text { Peto, Tunde; Blackwell's Queen's University Belfast, Centre for Public } \\
\text { Health } \\
\text { Cruise, Sharon; Queen's University Belfast School of Medicine Dentistry } \\
\text { and Biomedical Sciences, Centre for Public Health } \\
\text { McGuinness, Bernardette; Queen's University Belfast, Centre for Public } \\
\text { Health } \\
\text { Young, Ian } \\
\text { Kee, Frank } \\
\text { Azuara-Blanco, Augusto; Queen's University Belfast, Centre for Public } \\
\text { Health; Centre for Public Health, Queen's University Belfast }\end{array}$ \\
\hline Keywords: & $\begin{array}{l}\text { Glaucoma, Epidemiology, Intraocular pressure, Optic Nerve, Diagnostic } \\
\text { tests/Investigation }\end{array}$ \\
\hline
\end{tabular}

\section{SCHOLARONE Manuscripts}




\section{Title page}

Glaucoma in the Northern Ireland Cohort for the Longitudinal Study of Ageing (NICOLA): cohort profile, prevalence, awareness and associations

Paul McCann MBChB, PhD1, Ruth E. Hogg PhD'1, David M. Wright PhD1, Sara Pose-Bazarra $\mathrm{MD}^{2}$, Usha Chakravarthy $\mathrm{MD}, \mathrm{PhD}^{1,3}$, Tunde Peto $\mathrm{MD}$, $\mathrm{PhD}^{1,3}$, Sharon Cruise PhD ${ }^{1}$, Bernadette McGuinness MD, $\mathrm{PhD}^{1}$, Ian $\mathrm{S}$. Young MD1, Frank Kee MD1, Augusto Azuara-Blanco MD, PhD1

${ }^{1}$ Centre for Public Health, Queen's University Belfast, Belfast, UK

2University Hospital Complex of Ferrol, Ferro, Spain

${ }^{3}$ On behalf of the Network of Ophthalmic Reading Centres UK (NetwORC UK)

Corresponding author: Augusto Azuara-Blanco,

Address: Institute of Clinical Sciences, Block A, Royal Victoria Hospital, Belfast, BT12 6BA

Phone: 02890976430

Email: a.azuara-blanco@qub.ac.uk

Word Count: 4,376

Keywords: NICOLA, Glaucoma, Prevalence, Epidemiology

Paul McCann, pmccann45@qub.ac.uk, Institute of Clinical Sciences, Royal Victoria Hospital, Belfast, BT12 6BA

Ruth E. Hogg, r.e.hogg@qub.ac.uk, Institute of Clinical Sciences, Royal Victoria Hospital, Belfast, BT12 6BA

David M. Wright, d.wright@qub.ac.uk, Institute of Clinical Sciences, Royal Victoria Hospital, Belfast, BT12 6BA

Sara Pose-Bazarra, saraposeb@hotmail.com, University Hospital Complex of Ferrol, Ferro, Spain

Usha Chakravarthy, u.chakravarthy@qub.ac.uk, Institute of Clinical Sciences, Royal Victoria Hospital, Belfast, BT12 6BA

Tunde Peto, t.peto@qub.ac.uk, Institute of Clinical Sciences, Royal Victoria Hospital, Belfast, BT12 6BA

Sharon Cruise, s.cruise@qub.ac.uk, Institute of Clinical Sciences, Royal Victoria Hospital, Belfast, BT12 6BA 
Bernadette McGuinness, b.mcguiness@qub.ac.uk, Institute of Clinical Sciences, Royal Victoria Hospital, Belfast, BT12 6BA

Ian S. Young, i.young@qub.ac.uk, Institute of Clinical Sciences, Royal Victoria Hospital, Belfast, BT12 6BA

Frank Kee, f.kee@qub.ac.uk, Institute of Clinical Sciences, Royal Victoria Hospital, Belfast, BT12 6BA

Precis: The crude prevalence of glaucoma in Northern Ireland of $2.83 \%$ $(95 \% \mathrm{Cl} 2.31 \%, 3.46 \%)$ is comparable to other European population-based studies. Approximately two thirds of people with glaucoma were undiagnosed. Associations with glaucoma were consistent with current understanding of the disease. 


\begin{abstract}
Background/Aims: To describe the cohort profile of the Northern Ireland Cohort for the Longitudinal Study of Ageing (NICOLA) and to report the prevalence of, awareness of, and associations with glaucoma.

Methods: Using geographic stratification a representative sample of noninstitutionalised Northern Irish adults aged over 50 years was invited to participate. NICOLA participants underwent a computer assisted personal interview (CAPI), a self-completion questionnaire (SCQ) and a health assessment. The CAPI and SCQ collected comprehensive sociodemographic and health related data. At the health assessment participants underwent optic disc stereophotography, intraocular pressure (IOP) measurement using Ocular Response Analyser (ORA), autorefraction, spectral domain optical coherence tomography and self-reported history of glaucoma. We invited NICOLA participants suspected of having glaucoma due to optic disc appearance or raised IOP for clinical examination by a glaucoma expert and perimetry. Epidemiological definitions by the International Society Geographical and Epidemiological Ophthalmology were used to define glaucoma.
\end{abstract}

Results: Of 3,221 NICOLA participants (mean age 64.4, SD 8.5, female sex $51.7 \%$ ) who attended the health assessment component of the NICOLA study (and had a vertical cup to disc ratio measurement in at least one eye), 91 participants had glaucoma. Overall, the crude prevalence of glaucoma was $2.83 \%(95 \% \mathrm{Cl} 2.31 \%, 3.46 \%)$ and $67 \%$ of affected individuals did not give a self-reported history of glaucoma. 
Conclusions: The prevalence of glaucoma in Northern Ireland is comparable to other population-based studies of European populations. Approximately two thirds of people with glaucoma were undiagnosed. 


\section{INTRODUCTION}

There is a global trend for people to live longer and though welcome, this poses major challenges for economic, health and social policy. ${ }^{1}$ Longitudinal studies of older people aim to understand social, economic and biological factors that influence healthy ageing. ${ }^{2}$ The Northern Ireland Cohort for the Longitudinal Study of Ageing (NICOLA) is a large longitudinal study of ageing in Northern Ireland. ${ }^{3}$ It is a rich resource which enables the investigation of the biological, demographic and socioeconomic risk factors for glaucoma. Glaucoma, an age-related chronic eye disease, is the second leading cause of severe visual loss in the developed world and is therefore a major public health concern. ${ }^{4}$ The prevalence of glaucoma varies among ethnicities and regions. Europe has the lowest prevalence of glaucoma at $2.93 \%$ in people over 40.5

In 2002 the International Society Geographical and Epidemiological Ophthalmology (ISGEO) set guidelines to standardise case definitions of glaucoma for the purposes of prevalence studies. ${ }^{6}$ Since its introduction many studies have adopted the ISGEO guidelines, especially in Asia and Africa. ${ }^{6}$ At present, there is few data on the prevalence of glaucoma from Europe that is based on ISGEO criteria and this lack of knowledge makes comparisons between other ethnicities and geographic locations impossible. The NICOLA study performed high quality retinal imaging and obtained functional data therefore offers a unique opportunity to obtain robust estimates of glaucoma prevalence based on ISGEO definitions of glaucoma and to explore relevant risk factors in the European setting. 


\section{METHODS}

NICOLA is an ongoing longitudinal cohort study of ageing in a sample of the Northern Ireland population aged 50 years or older. The sampling strategy identified addresses within postcode-based geographic regions (geographic stratification). A fixed interval (systematic) sample was drawn from each postcode-based geographical stratum. ${ }^{2}$ The NICOLA assessment comprised of three elements; 1) a Computer Assisted Personal Interview (CAPI; Supplementary Table S1 in the online supplementary material) conducted at the participant's home by a trained interviewer, 2) a self-completion questionnaire (SCQ) that included the 9-item National Eye Institute Visual Function Questionnaire (NEI VFQ 9; Supplementary Table S2 in the online supplementary material), and, 3) a health assessment including a range of ophthalmic, anthropometric, cardiovascular, cognition and blood tests, which were performed at the Northern Ireland Clinical Research Facility (NICRF). The health assessment also included a detailed ophthalmic component during the visit to the NICRF. Data collection for the NICOLA study began in February 2014 and ended in 2018 . $^{2}$ Details of NICOLA study assessments can be found in Supplementary Methods 1 in the online supplementary material and at https://www.qub.ac.uk/sites/NICOLA/. Details of the ophthalmic component of the NICOLA study health assessment can also be found in Supplementary Methods 1 in the online supplementary material.

Vertical cup to disc ratio (VCDR) was used as the primary index of structural glaucomatous change following ISGEO recommendations. ${ }^{6}$ Details of VCDR grading and adjudication can be found in Supplementary Methods 2 in the online supplementary material. In brief, following a series of agreement 
analyses all discs graded with a VCDR $\geq 0.41$ by primary graders were regraded by an experienced grader (PMcC). All discs finally found to have a VCDR $\geq 0.7$ and/or VCDR asymmetry $\geq 0.2$ and/or disc haemorrhages and/or neuroretinal rim thinning or notching were re-graded (including fellow eyes) by a glaucoma expert (AAB). NICOLA participants who had a VCDR measurement in at least one eye were included in the analysis. NICOLA participants who did not attend the health assessment or who attended the health assessment but did not have a VCDR measurement were excluded.

NICOLA participants aged 50 years or older who attended the health assessment from the beginning of the study in February 2014 through $7^{\text {th }}$ January 2017 and had a VCDR $\geq 0.7$ and/or VCDR asymmetry $\geq 0.2$ and/or vertical neuroretinal rim ratio $(N R R R) \leq 0.1$ on optic disc stereophotography and/or intraocular pressure (IOP) $\geq 25 \mathrm{mmHg}$ on ORA tonometry (Goldmann correlated IOP (IOPg)) were invited for an additional examination by a glaucoma expert (AAB). Additional tests performed at this visit included visual field testing using Humphrey's Matrix frequency doubling technology (FDT) perimetry (Carl Zeiss Meditec Inc., Dublin, CA, USA) in low illumination. Instructions were provided, and a trial was performed in demonstration mode for 30 - 60 seconds to allow familiarisation with the test and clarification of the instructions. An initial 24-2 suprathreshold screening test was then performed followed by a 24-2 full threshold test. The 24-2 full threshold test was considered unreliable if there was $>33 \%$ for false positive errors, $>33 \%$ for false negative errors or $>33 \%$ for fixation errors. The $24-2$ full threshold test was repeated once if it was unreliable. Gonioscopy was performed at the slit lamp by the glaucoma expert. Pupil dilation (except in 
patients with narrow anterior chamber angle) was performed and biomicroscopy was carried out including optic disc examination.

ISGEO epidemiological definitions of glaucoma were used to define glaucoma and glaucoma related findings in this study (Supplementary Table S3 in the Supplementary material). ${ }^{6}$ The NICOLA study did not perform visual field tests on all participants thus the VCDR and IOP distributions of the subset of 'hypernormals' that are required to set the population-specific $97.5^{\text {th }}$ and $99.5^{\text {th }}$ percentiles could not be determined. Therefore, similar to other population-based studies, we set the VCDR $97.5^{\text {th }}$ and $99.5^{\text {th }}$ percentiles and the VCDR asymmetry $97.5^{\text {th }}$ and $99.5^{\text {th }}$ percentiles as 0.70 and 0.80 and 0.20 and 0.30 respectively. ${ }^{7,8}$

For ISGEO 1 classification, the Hodapp-Anderson-Parrish criteria (HAP), were applied to visual field tests and any of the following must have been present: 1) a Glaucoma Hemifield Test "outside normal limits" or, 2) a cluster of three or more points in a location typical for glaucoma, all of which are depressed on the pattern deviation plot at a $p<5 \%$ level and one of which is depressed at a $p<1 \%$ level or, 3 ) a pattern standard deviation that occurs in less than $5 \%$ of normal fields and, 4) visual field defect not explained by any other disease. If the visual field tests were unreliable or unavailable, participants were classified according to ISGEO 2 or ISGEO 3. ISGEO 2 classifications were only finalised after adjudication by a glaucoma expert $(A A B)$.

The presence of severe glaucoma was defined as a visual-field loss of either (1) mean deviation (MD) of $12 \mathrm{~dB}$ or worse or (2) absolute paracentral 
scotoma within the central $5^{\circ}$ of visual fields (defined as $\geq 1$ central $2 \times 2$ points on the pattern deviation plot). If visual fields were unreliable, the presence of severe disc damage (VCDR of more than or equal to 0.9 ) was used to define severe glaucoma. ${ }^{9}$

'Visual impairment' was defined using World Health Organisation (WHO) definitions. Blindness was defined in an eye if the presenting visual acuity was logMAR $>1.3$ (<3/60 Snellen) and moderate to severe visual impairment (MSVI) was defined in an eye if the presenting visual acuity was between $\log M A R 0.51$ and $\log M A R 1.3(<6 / 18, \geq 3 / 60$ Snellen).

This study was conducted according to the tenets of the Declaration of Helsinki. Ethical approval was obtained from the School of Medicine, Dentistry and Biomedical Sciences Ethics Committee, Queen's University Belfast and the Northern Ireland's Health and Social Care (HSC) Research Ethics Committee A (REC A) (REC reference: 16/NI/0247). We report the findings in accordance with the STROBE checklist. ${ }^{10}$

\section{Statistical analysis}

Summary statistics for baseline continuous variables and frequencies of categorical characteristics were reported for the cohort. Comparisons were performed between included and excluded participant using independent sample $t$ tests for parametric continuous variables and Mann-Whitney tests for non-parametric continuous variables. $\mathrm{X}^{2}$ or Fischer exact tests were used for categorical variables.

To determine if there were systematic differences between participants who attended the invitation for further clinical exam and testing and those who did 
not, comparisons of baseline demographic characteristics were performed. Independent sample $\mathrm{t}$ tests were used for parametric continuous variables and Mann-Whitney tests were used for non-parametric continuous variables. $\mathrm{X}^{2}$ or Fischer exact tests were used for categorical variables.

If a participant had glaucoma in at least one eye that person was defined as having glaucoma for prevalence purposes. If a participant had bilateral glaucoma, the participant was classified as glaucoma according to the eye with the highest level of evidence (i.e., ISGEO 1 > ISGEO 2 > ISGEO 3). Total and age specific prevalence of glaucoma and their $95 \%$ confidence intervals were calculated for the NICOLA sample $\geq 50$ years old. Crude and age-weighted prevalence were analysed. The age of participants at the time of the most recent visit to the NICOLA or clinical exam assessment was used for reporting prevalence estimates (age at ISGEO classification). Prevalence was calculated as the percentage of the number of persons with glaucoma to the total number of NICOLA participants who attended the NICOLA health assessment and had valid VCDR data available in $\geq 1$ eye. The methods and analysis of this study followed guidelines for evaluating prevalence studies published by Boyle et al ${ }^{11}$ and adapted for glaucoma.

To assess epidemiological associations with glaucoma, prevalent cases of glaucoma (by eye) were considered as the dependent variable in logistic regression analysis with Generalised Estimating Equations (GEE). The independent variables in the univariate analysis were identified based on comprehensive review of the literature and are described in Supplementary Methods 3 in the online supplementary material. 
In multivariable logistic regression analysis with GEE, we entered the variables age and sex (Model 1) then inserted independent variables from the univariate analysis that were statistically significant at the $p<0.05$ level. We then inserted all independent variables from Model 1 significant at the $p$ $<0.05$ level including age and sex and used reverse stepwise removal of variables by removing the least statistically significant at each step (Model 2). A $p$ value $<0.05$ was considered statistically significant.

Multivariable analysis was conducted using GEE which allowed data from both eyes and their intra-individual correlation to be considered. Subject variables were 'NICOLA participants' and within-subject variables were 'eyes'. Within-subject variables were eligible as independent variables within the multivariable analysis. Independent working correlation matrix was selected and the use of a robust estimator was selected to be robust against misspecification of the working correlation matrix.

Statistical analysis was performed using a software program (IBM SPSS Statistics for Windows, Version 24.0. Armonk, NY: IBM Corp) and R version 3.5.2 (R Foundation for Statistical Computing, Vienna, Austria). The precision of the prevalence estimate was calculated using methods described by Newcombe..$^{12}$ Prevalence was weighted using the Northern Ireland Statistics and Research Agency (NISRA) census 2011 and the 2017 population projection. ${ }^{13}$ Weighting was determined by using the package 'survey' in $\mathrm{R}$ version 3.5.2 (R Foundation for Statistical Computing, Vienna, Austria).

\section{RESULTS}

\section{NICOLA cohort profile}


The NICOLA study required 12,077 addresses (main sample) and in addition a $20 \%$ contingency (reserve sample) sample of 2,415 addresses. ${ }^{3}$ In total, 8,504 participants underwent NICOLA Wave 1 CAPI (response rate of 63\%).

${ }^{3}$ CAPI interviews were conducted on 8,504 of whom 3,221 participants attended the health assessment and had VCDR data available in $\geq 1$ eye (Figure 1). These 3,221 participants comprised the denominator for the prevalence estimate and were included in the association analysis. NICOLA participants with no VCDR data available in either eye were excluded from the prevalence denominator.

Baseline characteristics of the included 3,221 NICOLA participants were compared to the 5,044 excluded NICOLA participants (Table 1). Statistically significant differences in baseline demographics were found between included and excluded NICOLA participants. The numbers of missing VCDR, IOP and circumpapillary retinal nerve fibre layer (cRNFL) measurements are reported in Supplementary Table S4 in the online supplementary material.

In total, 229 NICOLA participants were initially considered to have been eligible for the additional examination by the glaucoma expert, of whom 49 were excluded based on specified criteria outlined in Figure 1. Invitations for clinical exam were sent to 180 NICOLA participants of whom 128 NICOLA attended for further clinical exam and visual field testing.

Baseline characteristics of 128 eligible NICOLA participants who attended the clinical examination were compared to the 101 NICOLA participants who did not attend (49 who were excluded from invitation plus 52 who declined 
invitation). Attendees were statistically significantly younger than nonattendees (Supplementary Table S5 in the online supplementary material). 
Table 1. Baseline characteristics of NICOLA participants and comparisons between 'Glaucoma in NICOLA participants' and 'NICOLA participants excluded from Glaucoma in NICOLA.'

\begin{tabular}{|c|c|c|c|c|}
\hline & $\begin{array}{l}\text { NICOLA } \\
\text { Cohort } \\
(n=8,265)\end{array}$ & $\begin{array}{l}\text { Included } \\
\text { participants } \\
(n=3,221)\end{array}$ & $\begin{array}{l}\text { Excluded } \\
\text { participants } \\
(\mathrm{n}=5,044) \\
\end{array}$ & $\mathrm{P}$ value $\dagger$ \\
\hline $\begin{array}{l}\text { Age (SD) at health } \\
\text { assessment } \\
(n=3,214)\end{array}$ & d.n.c. & $64.4(8.5)$ & d.n.c. & - \\
\hline $\begin{array}{l}\text { Age category at CAPI } \\
(n=8,257)\end{array}$ & & & & $<0.001$ \\
\hline $50-59(\%)$ & 2,778 & $\begin{array}{l}1,192 \\
(37.1)\end{array}$ & $1,586(31.4)$ & \\
\hline $60-69(\%)$ & 2,782 & $\begin{array}{l}1,246 \\
(38.8)\end{array}$ & $1,536(30.5)$ & \\
\hline $70-79(\%)$ & 1,850 & $633(19.7)$ & $1,217(24.1)$ & \\
\hline $80+(\%)$ & 847 & $143(4.4)$ & $704(14.0)$ & \\
\hline $\begin{array}{l}\text { Sex } \\
(n=8,257)\end{array}$ & & & & $<0.001$ \\
\hline Male (\%) & 3,738 & $\begin{array}{l}1,552 \\
(48.3)\end{array}$ & $2,186(43.3)$ & \\
\hline Female (\%) & 4,519 & $\begin{array}{l}1,662 \\
(51.7)\end{array}$ & $2,857(56.7)$ & \\
\hline $\begin{array}{l}\text { Habitation } \\
(\mathrm{n}=6,495)\end{array}$ & & & & 0.023 \\
\hline $\begin{array}{l}\text { Urban / city / } \\
\text { townland (\%) }\end{array}$ & 4,443 & $\begin{array}{l}1,669 \\
(70.1)\end{array}$ & $2,774(67.4)$ & \\
\hline Rural (\%) & 2,052 & $711(29.9)$ & $1,341(32.6)$ & \\
\hline $\begin{array}{l}\text { Education level } \\
(n=8,182)\end{array}$ & & & & $<0.001$ \\
\hline None (\%) & 292 & $68(2.1)$ & $224(4.5)$ & \\
\hline $\begin{array}{l}\text { Some primary } \\
\text { (not complete) } \\
(\%)\end{array}$ & 151 & $30(0.9)$ & $121(2.4)$ & \\
\hline $\begin{array}{l}\text { Primary or } \\
\text { equivalent (\%) }\end{array}$ & 1,651 & $367(11.4)$ & $1,284(25.8)$ & \\
\hline $\begin{array}{l}\text { GCSE / O level } \\
\text { or equivalent } \\
(\%)\end{array}$ & 3,021 & $\begin{array}{l}1,136 \\
(35.4)\end{array}$ & $1,885(37.9)$ & \\
\hline $\begin{array}{l}\text { A level or } \\
\text { equivalent (\%) }\end{array}$ & 608 & $268(8.3)$ & $340(6.8)$ & \\
\hline $\begin{array}{l}\text { Diploma } \\
\text { certificate or } \\
\text { equivalent (\%) } \\
\end{array}$ & 1,154 & $581(18.1)$ & $573(11.5)$ & \\
\hline $\begin{array}{l}\text { Undergraduate } \\
\text { primary degree } \\
(\%)\end{array}$ & 644 & $372(11.6)$ & $272(5.5)$ & \\
\hline
\end{tabular}




\begin{tabular}{|c|c|c|c|c|}
\hline $\begin{array}{l}\text { Postgraduate / } \\
\text { higher degree } \\
(\%)\end{array}$ & 661 & $389(12.1)$ & $272(5.5)$ & \\
\hline $\begin{array}{l}\text { Siblings with glaucoma } \\
(n=7,743)\end{array}$ & & & & 0.054 \\
\hline No $(\%)$ & 7,464 & \begin{tabular}{|l|}
2,943 \\
$(96.9)$ \\
\end{tabular} & $4,521(96.1)$ & \\
\hline Yes (\%) & 279 & $94(3.1)$ & $185(3.9)$ & \\
\hline $\begin{array}{l}\text { Past smoking status } \\
(\mathrm{n}=8,170)\end{array}$ & & & & $<0.001$ \\
\hline Never (\%) & 3,953 & $\begin{array}{l}1,687 \\
(52.5)\end{array}$ & $2,266(45.7)$ & \\
\hline Ever (\%) & 4,217 & $\begin{array}{l}1,524 \\
(47.5)\end{array}$ & $2,693(54.3)$ & \\
\hline $\begin{array}{l}\text { Current smoking } \\
\text { status } \\
(\mathrm{n}=4,217)\end{array}$ & & & & $<0.001$ \\
\hline Ex-smoker (\%) & 2,863 & $\begin{array}{l}1,210 \\
(79.4)\end{array}$ & $1,653(61.4)$ & \\
\hline $\begin{array}{l}\text { Current smoker } \\
(\%)\end{array}$ & 1,354 & $314(20.6)$ & $1,040(38.6)$ & \\
\hline $\begin{array}{l}\text { Self-report } \\
\text { cardiovascular disease } \\
\text { (Angina or MI) } \\
(\mathrm{n}=8,191)\end{array}$ & 80 & & & $<0.001$ \\
\hline Yes $(\%)$ & 2,890 & $993(30.9)$ & $1,897(38.1)$ & \\
\hline No (\%) & 5,301 & $\begin{array}{l}2,220 \\
(69.1)\end{array}$ & $3,081(61.9)$ & \\
\hline $\begin{array}{l}\text { Self-report } \\
\text { cerebrovascular } \\
\text { disease (Stroke or } \\
\text { TIA) } \\
(n=8,257)\end{array}$ & & & & $<0.001$ \\
\hline Yes (\%) & 491 & $131(4.1)$ & $360(7.1)$ & \\
\hline No (\%) & 7,766 & $\begin{array}{l}3,083 \\
(95.9) \\
\end{array}$ & $4,683(92.9)$ & \\
\hline $\begin{array}{l}\text { Self-report } \\
\text { Hypertension } \\
(\mathrm{n}=8,257)\end{array}$ & & & & $<0.001$ \\
\hline Yes (\%) & 3,337 & $\begin{array}{l}1,132 \\
(35.2) \\
\end{array}$ & $2,838(56.3)$ & \\
\hline No (\%) & 4,920 & $\begin{array}{l}2,082 \\
(64.8) \\
\end{array}$ & $2,205(43.7)$ & \\
\hline $\begin{array}{l}\text { Self-report High } \\
\text { cholesterol } \\
(n=8,257)\end{array}$ & & & & 0.403 \\
\hline Yes $(\%)$ & 2,996 & $\begin{array}{l}1,184 \\
(36.8)\end{array}$ & $1,812(35.9)$ & \\
\hline
\end{tabular}




\begin{tabular}{|c|c|c|c|c|}
\hline No (\%) & 5,261 & $\begin{array}{l}2,030 \\
(63.2)\end{array}$ & $3,231(64.1)$ & \\
\hline $\begin{array}{l}\text { Self-report Diabetes } \\
\text { mellitus } \\
(n=8,257)\end{array}$ & & & & $<0.001$ \\
\hline Yes $(\%)$ & 926 & $255(7.9)$ & $671(13.3)$ & \\
\hline No $(\%)$ & 7,331 & $\begin{array}{l}2,959 \\
(92.1)\end{array}$ & $4,372(86.7)$ & \\
\hline $\begin{array}{l}\text { Self-report Parkinson's } \\
\text { disease } \\
(\mathrm{n}=8,257)\end{array}$ & & & & 0.727 \\
\hline Yes (\%) & 28 & $10(0.3)$ & $18(0.4)$ & \\
\hline No (\%) & 8,229 & $\begin{array}{l}3,204 \\
(99.7)\end{array}$ & $5,025(99.6)$ & \\
\hline $\begin{array}{l}\text { Median income } \\
\text { domain score (IQR) } \\
(n=8,246)\end{array}$ & $\begin{array}{l}0.2000 \\
(0.1300 \\
0.3000)\end{array}$ & $\begin{array}{l}0.1800 \\
(0.1100 \\
0.2700)\end{array}$ & $\begin{array}{l}0.2100 \\
(0.1400 \\
0.3200)\end{array}$ & $<0.001 \mp$ \\
\hline $\begin{array}{l}\text { Median house value } \\
(\text { IQR) } \\
(\mathrm{n}=8,085)\end{array}$ & $\begin{array}{l}115000 \\
(77500 \\
160000)\end{array}$ & $\begin{array}{l}135000 \\
(95000 \\
185000)\end{array}$ & $\begin{array}{l}100000 \\
(72500 \\
145000)\end{array}$ & $<0.001 \mp$ \\
\hline $\begin{array}{l}\text { Median NEI VFQ } 9 \\
\text { score (IQR) } \\
(n=4,067)\end{array}$ & $\begin{array}{l}12.00 \\
(10.00 \\
15.00)\end{array}$ & $\begin{array}{l}12.00 \\
(10.00 \\
15.00)\end{array}$ & $\begin{array}{l}13.00(10.00 \\
16.00)\end{array}$ & 0.002 \\
\hline
\end{tabular}

$\dagger$ Included participants compared to excluded participants; $¥$ Mann Whitney U test; d.n.c. data not collected

\section{Glaucoma prevalence}

In total, there were 91 participants diagnosed with glaucoma. The crude prevalence of glaucoma was $2.83 \%(95 \% \mathrm{Cl} 2.31 \%, 3.46 \%) .{ }^{12}$ The population age-weighted prevalence of glaucoma was $3.19 \%(95 \% \mathrm{Cl} 2.55$, $3.97)$ for the 2011 population census and $3.24 \%(95 \% \mathrm{Cl} 2.59,4.04)$ for the 2017 population projection. The age-stratified crude and age-weighted prevalence of glaucoma are presented in Figure 2 and Table 2. Two thirds $(67 \% ; 61 / 91)$ of participants with glaucoma did not self-report a history of glaucoma and $1.37 \%(43 / 3,130)$ of participants without glaucoma selfreported a history of glaucoma.

\section{Glaucoma sub-types and severity}


For the 91 NICOLA participants with glaucoma, glaucoma sub-types were reported for the 128 participants who attended clinical examination and are shown in Supplementary Table S6 in the online supplementary material. Of the 128 participants who attended clinical examination here were 36 right eyes and 36 left eyes from 48 participants diagnosed with POAG and 4 right eyes and 7 left eyes from 7 participants with PACG. One of these participants had one eye with POAG and one eye with PACG. There was 1 eye from 1 participant with pseudo-exfoliation glaucoma and both eyes from 1 participant with pigmentary glaucoma. There were 20 right eyes and 15 left eyes from 31 participants with severe glaucoma and 8 participants had severe glaucoma in both eyes. 
Table 2. Age stratified and total, crude prevalence, 2011 census age-weighted prevalence and 2017 population projection ageweighted prevalence

\begin{tabular}{|l|l|l|l|l|l|l|}
\hline Age group & $\begin{array}{l}\text { Age-stratified } \\
\text { crude } \\
\text { prevalence }\end{array}$ & $\begin{array}{l}\text { Age-stratified } \\
\text { crude prevalence } \\
95 \% \text { Cls }\end{array}$ & $\begin{array}{l}\text { 2011 census age- } \\
\text { weighted } \\
\text { prevalence }\end{array}$ & $\begin{array}{l}\text { 2011 census age- } \\
\text { weighted } \\
\text { prevalence } \\
95 \% \text { Cls }\end{array}$ & $\begin{array}{l}\text { 2017 population } \\
\text { projection age- } \\
\text { weighted } \\
\text { prevalence }\end{array}$ & $\begin{array}{l}\text { 2017 population } \\
\text { projection age- } \\
\text { weighted } \\
\text { prevalence } \\
95 \% \text { Cls }\end{array}$ \\
\hline $50-59$ & 1.13 & $0.64,1.97$ & 1.28 & $0.73,2.25$ & 1.29 & $0.74,2.27$ \\
\hline $60-69$ & 2.22 & $1.54,3.20$ & 1.73 & $1.20,2.50$ & 1.62 & $1.12,2.34$ \\
\hline $70-79$ & 4.78 & $3.43,6.61$ & 4.30 & $3.09,5.95$ & 4.49 & $3.22,6.22$ \\
\hline $80+$ & 9.60 & $6.03,14.95$ & 20.55 & $12.91,32.00$ & 21.32 & $13.40,33.19$ \\
\hline Total & 2.83 & $2.31,3.47$ & 3.19 & $2.55,3.97$ & 3.24 & \\
\hline
\end{tabular}




\section{ISGEO classifications by participant and by eye}

The number of eyes diagnosed with glaucoma classified by ISGEO categories and laterality is shown in Supplementary Table S7 in the online supplementary material. 52 participants were diagnosed with glaucoma according to ISGEO category 1 and 39 participants were diagnosed with glaucoma according to ISGEO category 2.

In 23 participants $(18.0 \%, 23$ / 128) visual field tests were either unreliable or the abnormality was deemed to be due to causes other than glaucoma (Supplementary Table S8 in the online supplementary material). Data from eyes in this group were eligible for ISGEO category 2 classification in prevalence calculations using the optic disc findings.

\section{Visual Impairment in Glaucoma}

There was 1 right eye with glaucoma that was blind according to WHO criteria, however, glaucoma was not the primary cause of blindness. There were 3 left eyes with glaucoma that were visually impaired according to WHO criteria, and the remaining glaucomatous eyes did not have visual impairment according to WHO criteria.

\section{Other glaucoma related findings}

Of the 130 eyes with glaucoma, 17 had IOPg $>21 \mathrm{mmHg}$. In total, 34 right eyes and 40 left eyes were disc suspects. Of the 34 right eye disc suspects, 5 had IOPg $>21 \mathrm{mmHg}$. Of the 40 left eye disc suspects, 5 had IOPg $>21$ $\mathrm{mmHg}$ and 2 had IOPg missing. 
Among the 3,221 included NICOLA participants there were 265 participants $(8.2 \%)$ with ocular hypertension ( $>21 \mathrm{mmHg}$ in $\geq 1$ eye) according to IOPg and there were 82 participants $(2.6 \%)$ with $\mathrm{IOPg} \geq 24 \mathrm{mmHg}$ in $\geq 1$ eye at the NICOLA health assessment. There were 327 participants (10.2\%) with ocular hypertension ( $>21 \mathrm{mmHg}$ in $\geq 1$ eye) according to IOPcc and there were 109 participants $(3.4 \%)$ with IOPcc $\geq 24 \mathrm{mmHg}$ in $\geq 1$ eye at the NICOLA health assessment.

\section{Associations between glaucoma and systemic, ocular and sociodemographic factors}

Univariate and multivariable analysis models for associations with glaucoma are reported in Table 3 . In the fully adjusted multivariable model, older age, increased IOPg and increased NEI VFQ 9 question 2 subscale score (how much time do you worry about your eyesight?) were associated with increased odds ratio of having glaucoma. Increased corneal hysteresis $(\mathrm{CH})$ and hyperopic increase in spherical equivalent were associated with reduced odds ratio of eyes having glaucoma. Decreased mean global cRNFL thickness was associated with significantly higher odds of glaucoma when adjusted for age and sex. 
Table 3. Univariate and multivariate analysis of associations with glaucomatous eyes.

\begin{tabular}{|c|c|c|c|c|c|c|c|c|c|}
\hline & \multicolumn{3}{|c|}{ Univariate } & \multicolumn{3}{|c|}{ Multivariate Model $1 \mathrm{k}$} & \multicolumn{3}{|c|}{ Multivariate Model $2^{\circ}$} \\
\hline & $\begin{array}{l}\text { OR } \\
(\operatorname{Exp}(\mathrm{B}))\end{array}$ & $95 \% \mathrm{Cl}$ & $P$ value & $\begin{array}{l}\text { OR } \\
(\operatorname{Exp}(B))\end{array}$ & $95 \% \mathrm{Cl}$ & $P$ value & $\begin{array}{l}\text { OR } \\
(\operatorname{Exp}(B))\end{array}$ & $95 \% \mathrm{Cl}$ & $P$ value \\
\hline Age at ISGEO classification & 1.080 & $\begin{array}{l}1.054 \\
1.107\end{array}$ & $<0.001$ & 1.093 & $\begin{array}{l}1.067 \\
1.121\end{array}$ & $<0.001$ l,n & 1.097 & $\begin{array}{l}1.062 \\
1.132 \\
\end{array}$ & $<0.001$ \\
\hline $50-59$ & Ref & & & & & & & & \\
\hline $60-69$ & 2.026 & $\begin{array}{l}0.987 \\
4.162\end{array}$ & 0.054 & 2.015 & $\begin{array}{l}0.980 \\
4.141\end{array}$ & $0.057^{\prime}$ & - & - & - \\
\hline $70-79$ & 4.613 & $\begin{array}{l}2.288 \\
9,303\end{array}$ & $<0.001$ & 4.548 & $\begin{array}{l}2.244 \\
9.216\end{array}$ & $<0.001$ & - & - & - \\
\hline $80+$ & 11.359 & $\begin{array}{l}5.151 \\
25.046 \\
\end{array}$ & $<0.001$ & 11.109 & $\begin{array}{l}4.972 \\
24.818 \\
\end{array}$ & $<0.001$ & - & - & - \\
\hline Sex & & & & + & & & & & \\
\hline Female & Ref & & 8 & 2 & & & & & \\
\hline Male & 1.337 & $\begin{array}{l}0.862 \\
2.073\end{array}$ & 0.194 & 1.152 & $\begin{array}{l}0.736 \\
1.802 \\
\end{array}$ & $0.536^{m}$ & - & - & - \\
\hline Eye & & & & + & 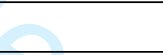 & & & & \\
\hline Right & Ref & & & & +1 & & & & \\
\hline Left & 1.134 & $\begin{array}{l}0.908 \\
1.416\end{array}$ & 0.267 & - & - & - & - & - & - \\
\hline Dwelling & & & & & & 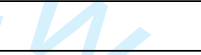 & & & \\
\hline Urban & Ref & & & & & 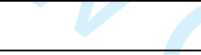 & 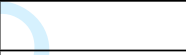 & & \\
\hline Rural & 0.854 & $\begin{array}{l}0.487 \\
1.497\end{array}$ & 0.581 & - & - & - & - & - & - \\
\hline Smoking status & & & & & & & & _ & \\
\hline Never & Ref & & & & & & & & \\
\hline
\end{tabular}




\begin{tabular}{|c|c|c|c|c|c|c|c|c|c|}
\hline Ever & 0.918 & $\begin{array}{l}0.592 \\
1.424\end{array}$ & 0.703 & - & - & - & - & - & - \\
\hline Ex-smoker & Ref & & & & & & & & \\
\hline Current & 0.520 & $\begin{array}{l}0.239 \\
1.128\end{array}$ & 0.098 & - & - & - & - & - & - \\
\hline Never & Ref & & & & & & & & \\
\hline Current & 0.646 & $\begin{array}{l}-0.154 \\
1.445\end{array}$ & 0.114 & - & - & - & - & - & - \\
\hline Sibling history of glaucoma & 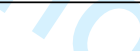 & & & & & & & & \\
\hline No & Ref & _ & & & & & & & \\
\hline Yes & 3.325 & $\begin{array}{l}1.512 \\
7.311 \\
\end{array}$ & 0.003 & 2.986 & $\begin{array}{l}1.358 \\
6.565\end{array}$ & $0.006^{n}$ & - & - & - \\
\hline Number of falls in past year & & 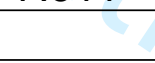 & 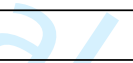 & & & & & & \\
\hline$<2$ & Ref & & 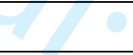 & 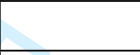 & & & & & \\
\hline$\geq 2$ & 1.617 & $\begin{array}{l}0.685 \\
3.814\end{array}$ & 0.273 & - & - & - & - & - & - \\
\hline Educational attainment & & & & 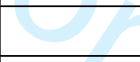 & & & & & \\
\hline $\begin{array}{l}\text { None or primary } \\
\text { incomplete }\end{array}$ & Ref & & & & & & & & \\
\hline Primary equivalent & 1.001 & $\begin{array}{l}0.253 \\
3.966\end{array}$ & 0.998 & - & - & - & - & - & - \\
\hline GCSE equivalent & 0.882 & $\begin{array}{l}0.247 \\
3.144\end{array}$ & 0.847 & - & - & - & - & - & - \\
\hline A level equivalent & 1.287 & $\begin{array}{l}0.329 \\
5.032\end{array}$ & 0.716 & - & - & - & - & - & - \\
\hline $\begin{array}{l}\text { Diploma certificate } \\
\text { equivalent }\end{array}$ & 1.229 & $\begin{array}{l}0.336 \\
4.489\end{array}$ & 0.756 & - & - & - & - & - & - \\
\hline Primary degree & 0.854 & $\begin{array}{l}0.208 \\
3.500\end{array}$ & 0.826 & - & - & - & - & - & - \\
\hline
\end{tabular}




\begin{tabular}{|c|c|c|c|c|c|c|c|c|c|}
\hline Postgraduate degree & 0.880 & $\begin{array}{l}0.225 \\
3.436\end{array}$ & 0.854 & - & - & - & - & - & - \\
\hline Hypertension & 1.400 & $\begin{array}{l}0.882 \\
2.222\end{array}$ & 0.154 & - & - & - & - & - & - \\
\hline Diabetes Mellitus & 1.357 & $\begin{array}{l}0.751 \\
2.450\end{array}$ & 0.312 & - & - & - & - & - & - \\
\hline High cholesterol & 1.137 & $\begin{array}{l}0.714 \\
1.811 \\
\end{array}$ & 0.589 & - & - & - & - & - & - \\
\hline Parkinson's disease & 1.387 & $\begin{array}{l}0.193 \\
9.962\end{array}$ & 0.745 & - & - & - & - & - & - \\
\hline $\begin{array}{l}\text { Cardiovascular disease (self- } \\
\text { reported angina or } \mathrm{MI} \text { ) }\end{array}$ & 1.504 & $\begin{array}{l}0.962 \\
2.350\end{array}$ & 0.073 & - & - & - & - & - & - \\
\hline $\begin{array}{l}\text { Cerebrovascular disease } \\
\text { (self-reported stroke or TIA) }\end{array}$ & 2.699 & $\begin{array}{l}1.286 \\
5.664\end{array}$ & 0.009 & 1.673 & $\begin{array}{l}0.745 \\
3.758\end{array}$ & 0.212 & - & - & - \\
\hline Cataract surgery & 2.605 & $\begin{array}{l}1.335 \\
5.084\end{array}$ & 0.005 & 1.820 & $\begin{array}{l}0.879 \\
3.768\end{array}$ & 0.107 & - & - & - \\
\hline $\mathrm{BMI}$ & & & & +8 & & & & & \\
\hline Underweight $(<18.5)$ & $\mathrm{a}$ & $\mathrm{a}$ & $\mathrm{a}$ & - & - & - & - & - & - \\
\hline Normal (18.5-24.9) & Ref & & & & $\bar{a}$ & & & & \\
\hline Overweight (25-29.9) & 1.043 & $\begin{array}{l}0.578 \\
1.882\end{array}$ & 0.889 & - & - & - & - & - & - \\
\hline Obese $(\geq 30)$ & 1.002 & $\begin{array}{l}0.541 \\
1.855\end{array}$ & 0.994 & - & - & - & - & - & - \\
\hline Height (per cm) & 1.004 & $\begin{array}{l}0.979 \\
1.029\end{array}$ & 0.769 & - & - & - & - & - & - \\
\hline Weight (per kg) & 0.998 & $\begin{array}{l}0.984 \\
1.012\end{array}$ & 0.776 & - & - & - & - & - & - \\
\hline Waist (per cm) & 1.006 & $\begin{array}{l}0.991 \\
1.022\end{array}$ & 0.434 & - & - & - & - & - & - \\
\hline
\end{tabular}




\begin{tabular}{|c|c|c|c|c|c|c|c|c|c|}
\hline Waist / hip ratio & 5.959 & $\begin{array}{l}0.445 \\
79.783\end{array}$ & 0.178 & - & - & - & - & - & - \\
\hline \multicolumn{10}{|l|}{ Hypertension stages } \\
\hline Normal & Ref & & & & & & & & \\
\hline Elevated & 1.073 & $\begin{array}{l}0.445 \\
2.584\end{array}$ & 0.876 & - & - & - & - & - & - \\
\hline Stage 1 & 1.145 & $\begin{array}{l}0.618 \\
2.122\end{array}$ & 0.668 & - & - & - & - & - & - \\
\hline Stage 2 & 1.086 & $\begin{array}{l}0.598 \\
1.971\end{array}$ & 0.786 & - & - & - & - & - & - \\
\hline $\begin{array}{l}\text { Spherical equivalent (per } \\
\text { dioptre) }\end{array}$ & 0.906 & $\begin{array}{l}0.853 \\
0.962\end{array}$ & 0.001 & 0.868 & $\begin{array}{l}0.816 \\
0.924\end{array}$ & $<0.001^{n}$ & 0.885 & $\begin{array}{l}0.827 \\
0.948 \\
\end{array}$ & $<0.001$ \\
\hline \multicolumn{10}{|l|}{ Refractive error } \\
\hline$>-0.5 \mathrm{D}$ & Ref & & 90 & & & & & & \\
\hline$-4.99 \mathrm{D}$ to $-0.5 \mathrm{D}$ & 1.487 & $\begin{array}{l}0.882 \\
2.508\end{array}$ & 0.137 & 1.672 & $\begin{array}{l}0.985 \\
2.839\end{array}$ & 0.057 & - & - & - \\
\hline$\leq-5 \mathrm{D}$ & 2.936 & $\begin{array}{l}1.316 \\
6.553\end{array}$ & 0.009 & 4.806 & $\begin{array}{l}2.142 \\
10.781\end{array}$ & $<0.001$ & - & - & - \\
\hline $\begin{array}{l}\text { Plasma cholesterol (per } \\
\mathrm{mmol} / \mathrm{L} \text { ) }\end{array}$ & 0.768 & $\begin{array}{l}0.644 \\
0.916\end{array}$ & 0.003 & 0.914 & $\begin{array}{l}0.757 \\
1.103\end{array}$ & 0.347 & - & - & - \\
\hline Plasma HDL (per mg/L) & 0.993 & $\begin{array}{l}0.980 \\
1.006\end{array}$ & 0.306 & - & - & - & - & - & - \\
\hline Plasma LDL (per mg/L) & 0.991 & $\begin{array}{l}0.985 \\
0.997\end{array}$ & 0.005 & 0.997 & $\begin{array}{l}0.991 \\
1.003\end{array}$ & 0.373 & - & - & - \\
\hline $\begin{array}{l}\text { Plasma triglycerides (per } \\
\mathrm{mg} / \mathrm{L} \text { ) }\end{array}$ & 0.998 & $\begin{array}{l}0.994 \\
1.001\end{array}$ & 0.216 & - & - & - & - & - & - \\
\hline Lipid lowering drugs & 1.528 & $\begin{array}{l}0.980 \\
2.383\end{array}$ & 0.062 & - & - & - & - & - & - \\
\hline
\end{tabular}




\begin{tabular}{|c|c|c|c|c|c|c|c|c|c|}
\hline Antihypertensive drugs & 2.231 & $\begin{array}{l}0.762 \\
6.535\end{array}$ & 0.143 & - & - & - & - & - & - \\
\hline Calcium channel blockers & 1.005 & $\begin{array}{l}0.346 \\
2.916\end{array}$ & 0.993 & - & - & - & - & - & - \\
\hline Beta-blocker drugs & 1.731 & $\begin{array}{l}1.009 \\
2.971\end{array}$ & 0.046 & 1.204 & $\begin{array}{l}0.696 \\
2.083\end{array}$ & 0.507 & - & - & - \\
\hline $\begin{array}{l}\text { Renin-angiotensin system } \\
\text { drugs }\end{array}$ & 1.243 & $\begin{array}{l}0.773 \\
1.998\end{array}$ & 0.369 & - & - & - & - & - & - \\
\hline Anti-Parkinsonian drugs & 1.571 & \begin{tabular}{|l|}
0.219 \\
11.277 \\
\end{tabular} & 0.654 & - & - & - & - & - & - \\
\hline Heart rate & 0.991 & $\begin{array}{l}0.972 \\
1.010 \\
\end{array}$ & 0.338 & - & - & - & - & - & - \\
\hline $\begin{array}{l}\text { Systolic blood pressure (per } \\
\mathrm{mmHg} \text { ) }\end{array}$ & 1.004 & \begin{tabular}{|l|}
0.993 \\
1.015 \\
\end{tabular} & 0.472 & - & - & - & - & - & - \\
\hline $\begin{array}{l}\text { Diastolic blood pressure (per } \\
\mathrm{mmHg} \text { ) }\end{array}$ & 0.995 & $\begin{array}{l}0.971 \\
1.019\end{array}$ & 0.662 & - & - & - & - & - & - \\
\hline IOPg (per mmHg) & 1.150 & $\begin{array}{l}1.094 \\
1.209\end{array}$ & $<0.001$ & 1.162 & $\begin{array}{l}1.100 \\
1.228\end{array}$ & $<0.001^{n}$ & 1.151 & $\begin{array}{l}1.074 \\
1.234\end{array}$ & $<0.001$ \\
\hline IOPcc (per mmHg) & 1.204 & $\begin{array}{l}1.143 \\
1.269\end{array}$ & $<0.001$ & 1.199 & $\begin{array}{l}1.131 \\
1.272\end{array}$ & $<0.001$ & - & - & - \\
\hline $\mathrm{CH}$ (per mmHg) & 0.584 & $\begin{array}{l}0.494 \\
0.689 \\
\end{array}$ & $<0.001$ & 0.650 & \begin{tabular}{|l|}
0.547 \\
0.773 \\
\end{tabular} & $<0.001^{n}$ & 0.726 & $\begin{array}{l}0.602 \\
0.876 \\
\end{array}$ & $<0.001$ \\
\hline CRF (per mmHg) & 0.911 & $\begin{array}{l}0.781 \\
1.061 \\
\end{array}$ & 0.231 & - & - & - & - & - & - \\
\hline \multicolumn{10}{|l|}{ Mean OPP } \\
\hline $1^{\text {st }}$ Quartile & 1.274 & $\begin{array}{l}0.680 \\
2.388\end{array}$ & 0.450 & - & - & - & - & - & - \\
\hline $2^{\text {nd }}$ Quartile & 1.210 & $\begin{array}{l}0.642 \\
2.281\end{array}$ & 0.555 & - & - & - & - & - & - \\
\hline
\end{tabular}




\begin{tabular}{|c|c|c|c|c|c|c|c|c|c|}
\hline $3^{\text {rd }}$ Quartile & 1.069 & \begin{tabular}{|l}
0.570 \\
2.003 \\
\end{tabular} & 0.836 & - & - & - & - & - & - \\
\hline $4^{\text {th }}$ Quartile & Ref & & & & & & & & \\
\hline \multicolumn{10}{|l|}{ Systolic OPP } \\
\hline $1^{\text {st }}$ Quartile & 0.950 & $\begin{array}{l}0.516 \\
1.749\end{array}$ & 0.869 & - & - & - & - & - & - \\
\hline $2^{\text {nd }}$ Quartile & 0.783 & $\begin{array}{l}0.403 \\
1.504\end{array}$ & 0.462 & - & - & - & - & - & - \\
\hline $3^{\text {rd }}$ Quartile & 1.071 & $\begin{array}{l}0.585 \\
1.959\end{array}$ & 0.824 & - & - & - & - & - & - \\
\hline $4^{\text {th }}$ Quartile & Ref & 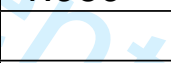 & & & & & & & \\
\hline \multicolumn{10}{|l|}{ Diastolic OPP } \\
\hline $1^{\text {st }}$ Quartile & 1.335 & \begin{tabular}{|l|}
0.744 \\
2.395
\end{tabular} & 0.333 & - & - & - & - & - & - \\
\hline $2^{\text {nd }}$ Quartile & 1.226 & $\begin{array}{l}0.682 \\
2.206\end{array}$ & 0.495 & - & - & - & - & - & - \\
\hline $3^{\text {rd }}$ Quartile & 0.526 & $\begin{array}{l}0.206 \\
1.064 \\
\end{array}$ & 0.074 & - & - & - & - & - & - \\
\hline $4^{\text {th }}$ Quartile & Ref & & & & 0 & & & & \\
\hline \multicolumn{10}{|l|}{ NEI-VFQ 9} \\
\hline Composite score & 1.043 & $\begin{array}{l}0.984 \\
1.106\end{array}$ & 0.152 & - & - & - & - & - & - \\
\hline Question 1 Scale ${ }^{b}$ & 1.152 & $\begin{array}{l}0.758 \\
1.750\end{array}$ & 0.509 & - & - & - & - & - & - \\
\hline Question 2 Scale $^{\mathrm{C}}$ & 1.401 & $\begin{array}{l}1.086 \\
1.808\end{array}$ & 0.009 & 1.153 & $\begin{array}{l}1.202, \\
1.980\end{array}$ & $0.001^{n}$ & 1.535 & $\begin{array}{l}1.158, \\
2.033\end{array}$ & 0.003 \\
\hline Question 3 Scale ${ }^{d}$ & 1.166 & $\begin{array}{l}0.875 \\
1.554\end{array}$ & 0.295 & - & - & - & - & - & - \\
\hline
\end{tabular}




\begin{tabular}{|c|c|c|c|c|c|c|c|c|c|}
\hline Question 4 Scale ${ }^{\mathrm{e}}$ & 1.064 & $\begin{array}{l}0.752 \\
1.506\end{array}$ & 0.727 & - & - & - & - & - & - \\
\hline Question 5 Scale ${ }^{f}$ & 1.675 & $\begin{array}{l}1.273 \\
2.204\end{array}$ & $<0.001$ & 1.494 & $\begin{array}{l}1.091 \\
2.046\end{array}$ & 0.012 & - & - & - \\
\hline Question 6 Scale 9 & 1.208 & $\begin{array}{l}0.725 \\
2.013 \\
\end{array}$ & 0.469 & - & - & - & - & - & - \\
\hline Question 7 Scale $^{h}$ & 1.213 & $\begin{array}{l}0.997 \\
1.475 \\
\end{array}$ & 0.054 & - & - & - & - & - & - \\
\hline Question 8 Scale $^{i}$ & 1.435 & $\begin{array}{l}1.019 \\
2.021\end{array}$ & 0.039 & 1.340 & $\begin{array}{l}0.893 \\
2.010\end{array}$ & 0.157 & - & - & - \\
\hline Question 9 Scale ${ }^{j}$ & 1.694 & $\begin{array}{l}1.206 \\
2.380 \\
\end{array}$ & 0.002 & 1.555 & $\begin{array}{l}1.074 \\
2.251\end{array}$ & 0.019 & - & - & - \\
\hline CES-D Depression Scale & 1.010 & $\begin{array}{l}0.983 \\
1.038 \\
\end{array}$ & 0.483 & - & - & - & - & - & - \\
\hline \multicolumn{10}{|l|}{$\begin{array}{l}\text { Visits to optician in past } 12 \\
\text { months }\end{array}$} \\
\hline None & Ref & & & +8 & & & & & \\
\hline$\geq 1$ visit & 1.324 & $\begin{array}{l}0.827 \\
2.121\end{array}$ & 0.243 & - & - & - & - & - & - \\
\hline $\begin{array}{l}\text { Overall income deprivation } \\
\text { domain score }\end{array}$ & 1.376 & $\begin{array}{l}0.294 \\
6.430 \\
\end{array}$ & 0.685 & - & - & - & - & - & - \\
\hline $\begin{array}{l}\text { Mean global cRNFL } \\
\text { thickness (per } \mu \mathrm{m} \text { increase) }\end{array}$ & 0.872 & $\begin{array}{l}0.854 \\
0.890 \\
\end{array}$ & $<0.001$ & 0.875 & $\begin{array}{l}0.858 \\
0.893\end{array}$ & $<0.001$ & - & - & - \\
\hline
\end{tabular}

a - Insufficient number of participants with BMI <18.5, b - At the present time would you say your eyesight is _ 1) excellent, 2) good,

3) fair, 4) poor, and 5) completely blind., c - How much time do you worry about your eyesight?, d - How much difficulty do you have reading ordinary print newspaper?, e - How much difficulty do you have doing work or hobbies that require you to see well up close such as cooking, sewing, fixing things around the house or using hand tools?, $f$ - Because of your eyesight how much difficulty do 
you have going down steps, stairs or curbs in dim light or at night?, g - How much difficulty do you have driving during daytime in familiar places?, h - Are you limited in how long you can walk or other activities such as housework, childcare, school or community activities because of your eyesight?, i - Because of your eyesight how much difficulty do you have noticing objects off to the side while you are walking along?, j - Because of your eyesight how much difficulty do you have finding something off a crowded shelf?, k - Age and sex adjusted model, , I - adjusted by Sex, m - adjusted by Age at ISGEO classification, $\mathrm{n}$ - Variables which were entered into the multivariate model for stepwise regression, o - Fully adjusted model, OR - odds ratio, $95 \% \mathrm{Cl}-95 \%$ confidence interval 


\section{DISCUSSION}

The NICOLA study is a population-based longitudinal study of ageing in Northern Ireland. In this study we investigated the prevalence of, awareness of and determinants of glaucoma. We found that glaucoma was prevalent in $2.83 \%(95 \% \mathrm{Cl}$ $2.31 \%, 3.46 \%$ ) of the population aged 50 years and older (crude prevalence). The population age-weighted prevalence was $3.19 \%(95 \% \mathrm{Cl} 2.55,3.97)$ for the 2011 population census and $3.24 \%(95 \% \mathrm{Cl} 2.59,4.04)$ for the 2017 population projection. Age-stratified crude prevalence showed that prevalence increased from 1.13\% $(95 \% \mathrm{Cl} 0.64,1.97)$ in the $50-59$ year old age group to $9.60 \%(95 \% \mathrm{Cl} 6.03,14.95)$ in the $80+$ age group. Our results are comparable to pooled estimations of glaucoma prevalence in European populations in the age range $40-80$ years $(2.93 \%, 95 \% \mathrm{Cl}$ $1.85 \%, 4.40 \%) .^{5}$

The NICOLA study provides a further estimation of glaucoma prevalence within a European population. There have been few other studies of European populations that have reported the prevalence of glaucoma using ISGEO definitions. Recently, the Gutenberg Health Study reported that the prevalence of glaucoma (of all subtypes) was $1.44 \%$, however the population included, spanned an age range from 35 to 74 years which is considerably younger than the present study. The EPIC-Norfolk eye study, which used a multistage clinical assessment, reported that the prevalence of glaucoma (of all sub-types) was $4.2 \%(95 \% \mathrm{Cl} 3.8 \%, 4.6 \%)$ in a sample of 8,623 participants aged 48-92 years in Norfolk, England. ${ }^{14}$ This is somewhat higher than the population age-weighted prevalence of $3.19 \%$ that we observed in NICOLA. The reasons for the difference in prevalence between NICOLA and EPIC-Norfolk eye study may be due to differences in methodology or population factors. Furthermore, it represented a third wave (second wave follow up) study of the EPIC cohort which 
may have been subject to attrition and selection bias. The Reykjavik eye study reported the prevalence of glaucoma (OAG including pseudoexfoliation glaucoma) to be $4.0 \%$ in participants aged 50 years and older however it had a relatively small sample of 1,045 participants. ${ }^{8}$ The ALIENOR study reported the prevalence of glaucoma (OAG) to be $4.8 \%$ in 944 French subjects however it only investigated participants aged 73 years and older. ${ }^{15}$

The UK-Biobank study which enrolled participants aged 40 to 69 years, had a prevalence of $1.7 \%$ based on self-report and those reporting glaucoma were more likely to be older and male. ${ }^{16}$ The findings of the UK-Biobank study are unlikely to be a true representation of glaucoma prevalence due to recall bias. Furthermore the response rate for the UK Biobank study was low (5.5\%) which is likely to impact on its representativeness of the general population.

The prevalence of glaucoma in US populations of predominantly white ethnicity has been reported to range between $1.29 \%$ to $2.1 \%,{ }^{17-22}$ however comparisons with these studies that were conducted several decades ago is confounded as the definition of glaucoma has evolved. ${ }^{19,20,22}$ It is recognised that even minor differences in the definition of glaucoma can cause wide variations in prevalence estimates. ${ }^{23}$ While there are contemporary US-based studies, these have used monoscopic optic disc photographs without full-threshold visual field testing for the diagnosis for glaucoma making direct comparisons with this study difficult. ${ }^{18,21}$ It is recognised that $50 \%$ of prevalent glaucoma is undiagnosed. ${ }^{24,25}$ In the NICOLA study two thirds of those with glaucoma were unaware of having the disease. This is a substantial level of under-ascertainment of glaucoma given the investment in primary eye care by the UK NHS, which provides free regular eye examinations to all 
people over 60 years of age, and also to people over 40 with family history of glaucoma. Our findings are consistent with those of the National Health and Nutrition Examination Survey (NHANES) 2005 - 2008 study in which $78 \%$ were previously undiagnosed ${ }^{21}$ and those of the Thessaloniki Eye Study where $57.1 \%$ and $34.9 \%$ of participants with POAG and pseudoexfoliation glaucoma respectively were previously undiagnosed. ${ }^{26}$ Similarly in the Singapore Epidemiology of Eye Diseases Study $72.1 \%$ of participants with primary glaucoma, were previously undiagnosed. ${ }^{27}$ The EPIC-Norfolk eye study reported that, of those who received a diagnosis of glaucoma, only $33.3 \%$ were previously unknown. It is unlikely that the difference in known glaucoma in the EPIC-Norfolk eye study compared to NICOLA was due to access to healthcare among participants, as the UK has a similar health care system. Differences may have arisen due to differences in sampling strategies as the EPIC-Norfolk eye study recruited participants with less deprivation than the general population.

The association analysis that we undertook revealed that the odds of glaucoma was greater with increased age, myopic decrease in spherical equivalent, increased IOP and decreased $\mathrm{CH}$. These findings are consistent with other study findings and current understanding of the disease. Glaucoma was not associated with gender in this study. Most glaucoma studies have identified a higher risk in men. Tham et al found an association with POAG ${ }^{28}$ and EPIC-Norfolk eye study observed a higher prevalence of glaucoma (of all sub-types) in males (5.2\%) compared to females (3.4\%). ${ }^{14}$ In the Korean National Health and Nutrition Examination Survey (KHANES) 2008 - 2011, male gender was associated with increased odds of glaucoma (OR 1.51, 95\%Cl 1.25, 1.83). ${ }^{29}$ 
An interesting finding in NICOLA was the higher odds of glaucoma after adjustment for age and sex with thinner mean global cRNFL thickness. Mean global CRNFL thickness was not entered into the final multivariable model due to significant collinearity with multiple other covariates associated with glaucoma. However additional risk factor models for cRNFL thickness are being constructed for reporting in future publications.

The NEI VFQ 9 has shown to have high reliability and validity regarding clinical measures of eye disease. ${ }^{30}$ We did not find an association between glaucoma diagnosis and NEI VFQ 9 composite score, however we observed an association with increased subscale score on question 2 (How much time do you worry about your eyesight?). NHANES 2005 - 2008 study also reported that glaucoma was not associated the NEI VFQ 9 composite score but they reported that glaucoma was associated with lower peripheral vision and distance vision subscale scores. ${ }^{31}$

We did not find an association between glaucoma and diabetes, hypertension, cerebrovascular disease, high cholesterol, systemic medication use, blood pressure or ocular perfusion pressure in the final multivariable model. These results are in agreement with some epidemiological studies but do not accord with others. ${ }^{32-36}$ Future analyses which incorporate electronic health record linkage may give a clearer estimation of associations between medical history, systemic medication use and glaucoma in the NICOLA study.

Overall income deprivation was not associated with glaucoma. Previous hospitalbased studies have reported that deprivation was significantly associated with late presentation of glaucoma. 9,37 The UK Biobank Study reported that self-reported glaucoma was associated with a higher mean Townsend deprivation index which 
indicates higher relative deprivation among those with self-reported glaucoma. ${ }^{38} \mathrm{We}$ did not find that glaucoma was associated with $\geq 2$ falls in the past year. This contrasts with findings from the Blue Mountains Eye Study and the Singapore Malay Eye study which found increased likelihood of falling in glaucoma. 39,40

Our study has a number of strength and weaknesses. It is one of the few European population-based studies to estimate glaucoma prevalence using the ISGEO definition and can therefore be used to make meaningful comparisons of glaucoma prevalence with other populations which also used ISGEO definition. ${ }^{14,41}$ The use of stereophotography to detect glaucomatous optic neuropathy in NICOLA is a major strength of the study compared to other modern studies which used nonstereoscopic optic disc pictures. ${ }^{7,18}$ Further strengths of the NICOLA study include the sampling strategy, large sample size, detailed ophthalmic phenotyping, meticulous ophthalmic data quality assurance, and the wide-ranging collection of sociodemographic and health related data.

Our study suffers from limitations and most notably, the rate of non-response at different levels of the recruitment process of the NICOLA study may have impacted on the generalizability of our results. The response rate for the NICOLA study was $63 \%$ however this is similar to other population-based studies from other countries. ${ }^{42}$ There were many statistically significant differences between excluded and included participants in our analysis of the NICOLA cohort and therefore the included participants are not representative of the cohort as a whole. Nonetheless our systematic sampling strategy and the population-based age-weighting are mitigating factors in the estimate of prevalence rates. Because the sample size in the NICOLA study was sufficiently large, we contend that the observed epidemiological associations are valid. ${ }^{43}$ 
Another limitation in NICOLA was the exclusion by individuals who were institutionalised therefore selection bias was introduced against the population who reside in nursing homes and other institutional accommodation who may be at risk of developing glaucoma due to their age and demographic profile.

Our study classified a relatively high number of participants as glaucoma using the ISGEO category 2 criteria $\left(43 \%, 39\right.$ / 91) in comparison to other studies. ${ }^{44}$ There was the potential for misclassification of large and anomalous discs as glaucomatous and for small glaucomatous optic discs to have gone undetected in this study. However a process of systematic adjudication with multiple measurements made of discs with VCDR $\geq 0.41$ aimed to reduce that possibility. Furthermore we used stereophotography which helps avoid misclassification of optic discs. Other studies have corrected VCDR measurements for disc size however this was not possible in the NICOLA study because biometry and keratometry data, which are required to calculate disc size on stereophotography, were not collected on NICOLA participants. ${ }^{41,45}$ In the NICOLA study it is possible that some of the included participants had stereophotographs missing for the 1 eye which was glaucomatous and this may have led to underestimation of the prevalence. We expect the impact of this limitation to be small.

A full description of the mechanisms of all glaucoma cases were not possible in this study because we were only able to enumerate primary and secondary open and closed angle glaucoma in the subgroup of 128 participants who attended for a full clinical examination. Therefore we are only able to reliably report the prevalence of glaucoma as a whole and are unable to comment on the prevalence of the respective subtypes of glaucoma in the NICOLA cohort. We did report that 31 participants had severe glaucoma in at least one eye. Further analysis of this data 
could investigate how many of these severe glaucoma cases were previously undiagnosed and the factors associated with severe glaucoma and the timeliness of its presentation. Previous studies have reported that socioeconomic deprivation is associated with more advanced glaucoma at presentation in newly diagnosed glaucoma patients. ${ }^{9}$

Although we were unable to perform perimetry on all participants it was performed in a subgroup of participants to confirm ISGEO category 1 classification of glaucoma. Similar methods to identify which participants should undergo perimetry have been used by other glaucoma prevalence studies, ${ }^{7,8,27,44}$ and it is likely that these provide higher quality evidence of glaucoma than studies which rely on only optic discs for all participants. ${ }^{18}$ Using perimetry in only a subset of participants, however, may be considered a weakness of the study. However, it is unlikely that performing visual field tests on a full-cohort of perimetry naïve participants would be an optimal method of detecting glaucoma. ${ }^{46}$ The use of ORA rather than GAT in the whole NICOLA population could be regarded as a potential weakness of the study because ORA IOP is not the current clinical reference standard, although ORA is less dependent on the skills of the examiner. Several population-based cohort studies including the EPIC-Norfolk eye study and UK Biobank study have used ORA tonometry. ${ }^{47}$

Although the ISGEO definition of glaucoma is a recognised international standard, it has several limitations. Vertical cup to disc ratios are a sensitive and simple quantitative anatomical indicator of glaucoma however, they are dependent on the size of the optic disc and have been reported to range from 0.00 to 0.85 in normal white populations. ${ }^{48}$ The use of percentile cut-offs to define glaucomatous optic neuropathy and glaucoma, could therefore lead to high numbers of false-negatives in 
small optic discs and high numbers of false-positives in large optic discs. ${ }^{49}$ We have tried to mitigate this limitation by re-grading all discs with VCDR $\geq 0.41$.

Currently the ISGEO criteria do not consider the additive value of modern imaging technologies such as SD-OCT to support the definitions. There are currently ongoing efforts to update the definition of glaucoma and the rich resource of data collected in the NICOLA study could be used to validate any new definitions. ${ }^{50}$

In summary, the NICOLA study is a population-based cohort study of ageing which provided detailed ophthalmic and glaucoma related phenotyping data. The estimated crude prevalence of glaucoma is $2.83 \%$ in the Northern Ireland population which is in agreement with pooled estimations of disease burden in European populations. Two thirds of people aged $\geq 50$ years old were unaware of having a diagnosis of glaucoma. These results have implications for implementation of primary health promotion and healthcare policy planning for glaucoma in the UK setting.

Acknowledgements: We are grateful to all the participants of the NICOLA Study, and the whole NICOLA team, which includes nursing staff, research scientists, clerical staff, computer and laboratory technicians, managers and receptionists. The Atlantic Philanthropies, the Economic and Social Research Council, the UKCRC Centre of Excellence for Public Health Northern Ireland, the Centre for Ageing Research and Development in Ireland, the Office of the First Minister and Deputy First Minister, the Health and Social Care Research and Development Division of the Public Health Agency, the Wellcome Trust/Wolfson Foundation and Queen's University Belfast provide core financial support for NICOLA. Belfast Association for the Blind provided core financial support for clinical examination. The authors alone are responsible for the interpretation of the data and any views or opinions presented 
are solely those of the authors and do not necessarily represent those of the NICOLA Study team.

Financial support: Atlantic Philanthropies, ESRC, HSC Research and Development, OFMDFM, UKCRC and Queen's University Belfast provide core financial support for NICOLA. Belfast Association for the Blind provided core financial support for clinical assessment of participants with possible glaucoma. The sponsors and funding organisations had no role in the design or conduct of this research. The sponsor or funding organisation had no role in the design or conduct of this research. No conflicting relationship exists for any author. 


\section{REFERENCES}

1. Christensen K, Doblhammer G, Rau R, Vaupel JW. Ageing populations: the challenges ahead. Lancet. 2009;374(9696):1196-1208. doi:10.1016/S01406736(09)61460-4

2. IALSA. Integrative Analysis of Longitudinal Studies of Aging and Dementia. https://www.maelstrom-research.org/mica/network/ialsa. Accessed August 4, 2019.

3. Burns F, Kee F. Methodology. In: Cruise S, Kee F, eds. Early Key Findings from a Study of Older People in Northern Ireland The NICOLA Study. ; 2017:89-97.

4. King A, Azuara-Blanco A, Tuulonen A. Glaucoma. BMJ. 2013;346:3518-3518. doi:10.1136/bmj.f3518

5. Tham YC, Li X, Wong TY, Quigley HA, Aung T, Cheng CY. Global prevalence of glaucoma and projections of glaucoma burden through 2040: A systematic review and meta-analysis. Ophthalmology. 2014;121(11):2081-2090. doi:10.1016/j.ophtha.2014.05.013

6. Foster PJ, Buhrmann R, Quigley HA, Johnson GJ. The definition and classification of glaucoma in prevalence surveys. Br J Ophthalmol. 2002;86:238-243. doi:10.1136/bjo.86.2.238

7. Delcourt C, Korobelnik J-F, Barberger-Gateau P, et al. Nutrition and agerelated eye diseases: The Alienor (Antioxydants, lipides essentiels, nutrition et maladies oculaires) study. J Nutr Health Aging. 2010;14(10):854-861. doi:10.1007/s12603-010-0131-9 
8. Jonasson F, Damji KF, Arnarsson A, et al. Prevalence of open-angle glaucoma in Iceland: Reykjavik eye study. Eye. 2003;17(6):747-753.

doi:10.1038/sj.eye. 6700374

9. Ng WS, Agarwal PK, Sidiki S, McKay L, Townend J, Azuara-Blanco A. The effect of socio-economic deprivation on severity of glaucoma at presentation. Br J Ophthalmol. 2010;94(1):85-87. doi:10.1136/bjo.2008.153312

10. von Elm E, Altman DG, Egger M, et al. The Strengthening the Reporting of Observational Studies in Epidemiology (STROBE) Statement: Guidelines for Reporting Observational Studies. PLOS Med. 2007;4(10):e296.

11. Boyle MH. Guidelines for evaluating prevalence studies. Evid Based Ment Health. 1998;1(2):37-39. doi:10.1136/ebmh.1.2.37

12. Newcombe R. Two-sided confidence intervals for the single proportion: comparison of seven methods. Stat Med. 1998;17:857-872.

13. Northern Ireland Statistics and Research Agency. http://www.ninis2.nisra.gov.uk/public/Theme.aspx. Accessed August 4, 2019.

14. Chan MPY, Broadway DC, Khawaja AP, et al. Glaucoma and intraocular pressure in EPIC-Norfolk Eye Study: cross sectional study. BMJ. 2017;358:j3889. doi:10.1136/bmj.j3889

15. Korobelnik JF, Delyfer MN, Rougier M, et al. Open-angle Glaucoma in French Elderly Subjects: The Alienor Study. Investig Ophthalmol Vis Sci. 2009;50:Eabstract 4078 .

16. Shweikh Y, Ko F, Chan MPY, et al. Measures of socioeconomic status and 
self-reported glaucoma in the UK Biobank cohort. Eye. 2015;29(10):13601367. doi:10.1038/eye.2015.157

17. Friedman DS, Wolfs RCW, O'Colmain BJ, et al. Prevalence of Open-Angle Glaucoma Among Adults in the United States. JAMA Ophthalmol. 2004;122(4):532-538. doi:10.1001/archopht.122.4.532

18. Gupta P, Zhao D, Guallar E, Ko F, Boland M V, Friedman DS. Prevalence of Glaucoma in the United States : The 2005 - 2008 National Health and Nutrition Examination Survey. Investig Ophthalmol Vis Sci. 2016;57:29052913. doi:10.1167/iovs.15-18469

19. Klein BEK, Klein R, Sponsel WE, et al. Prevalence of Glaucoma: The Beaver Dam Eye Study. Ophthalmology. 1992;99(10):1499-1504. doi:https://doi.org/10.1016/S0161-6420(92)31774-9

20. Kahn HA, Milton RC. Alternative Definitions of Open-Angle Glaucoma: Effect on Prevalence and Associations in the Framingham Eye Study. JAMA Ophthalmol. 1980;98(12):2172-2177. doi:10.1001/archopht.1980.01020041024003

21. Shaikh Y, Yu F, Coleman AL. Burden of Undetected and Untreated Glaucoma in the United States. Am J Ophthalmol. 2014;158(6):1121-1129.e1. doi:10.1016/j.ajo.2014.08.023

22. Tielsch JM, Sommer A, Katz J, Royall RM, Quigley HA, Javitt J. Racial Variations in the Prevalence of Primary Open-angle Glaucoma: The Baltimore Eye Survey. JAMA. 1991;266(3):369-374. doi:10.1001/jama.1991.03470030069026 
23. Wolfs RCW, Borger PH, Ramrattan RS, et al. Changing Views on Open-Angle Glaucoma : Definitions and Prevalences - The Rotterdam Study. Investig Ophthalmol Vis Sci. 2000;41:3309-3321.

24. Mitchell P, Smith W, Attebo K, Healey PR. Prevalence of Open-angle Glaucoma in Australia: The Blue Mountains Eye Study. Ophthalmology. 1996;103(10):1661-1669. doi:10.1016/S0161-6420(96)30449-1

25. Dielemans I, Vingerling JR, Wolfs RCW, Hofman A, Grobbee DE, de Jong PTVM. The Prevalence of Primary Open-angle Glaucoma in a Populationbased Study in The Netherlands: The Rotterdam Study. Ophthalmology. 1994;101(11):1851-1855. doi:10.1016/S0161-6420(94)31090-6

26. Topouzis F, Coleman AL, Harris A, et al. Factors Associated with Undiagnosed Open-Angle Glaucoma: The Thessaloniki Eye Study. Am J Ophthalmol. 2008;145(2):327-335.e1. doi:10.1016/j.ajo.2007.09.013

27. Chua J, Baskaran M, Ong PG, et al. Prevalence, Risk Factors, and Visual Features of Undiagnosed Glaucoma: The Singapore Epidemiology of Eye Diseases Study. JAMA Ophthalmol. 2015;133(8):938-946. doi:10.1001/jamaophthalmol.2015.1478

28. Kapetanakis V V, Chan MPY, Foster PJ, Cook DG, Owen CG, Rudnicka AR. Global variations and time trends in the prevalence of primary open angle glaucoma (POAG): a systematic review and meta-analysis. $\mathrm{Br} \mathrm{J}$ Ophthalmol. 2016;100(1):86-93. doi:10.1136/bjophthalmol-2015-307223

29. Kim KE, Kim MJ, Park KH, et al. Prevalence, Awareness, and Risk Factors of Primary Open-Angle Glaucoma: Korea National Health and Nutrition 
Examination Survey 2008-2011. Ophthalmology. 2016;123(3):532-541. doi:10.1016/j.ophtha.2015.11.004

30. Kodjebacheva G, Coleman AL, Ensrud KE, et al. Reliability and Validity of Abbreviated Surveys Derived from the National Eye Institute Visual Function Questionnaire: The Study of Osteoporotic Fractures. Am J Ophthalmol. 2010;149(2):330-340. doi:10.1016/j.ajo.2009.09.008

31. Lim AK, Shaikh Y, Yu F, Coleman AL. Quality of Life in Glaucoma as Measured by the Abbreviated National Eye Institute Visual Function Questionnaire. Investig Ophthalmol Vis Sci. 2011;52:5033.

32. Zhao D, Cho J, Kim MH, Friedman DS, Guallar E. Diabetes, fasting glucose, and the risk of glaucoma: A meta-analysis. Ophthalmology. 2015;122(1):72-78. doi:10.1016/j.ophtha.2014.07.051

33. Pertl L, Mossböck G, Wedrich A, et al. Triglycerides and Open Angle Glaucoma - A Meta-analysis with meta-regression. Sci Rep. 2017;7(1):7829. doi:10.1038/s41598-017-08295-1

34. Lee WJ, Jeoung JW, Na KI, Kim YK, Kim CY, Park KH. Relationship Between Open-angle Glaucoma and Stroke. J Glaucoma. 2018;27(1):22-27. doi:10.1097/IJG.0000000000000829

35. Hwang C, Lee YJ, Bae JH. A meta-analysis of glaucoma risk in hyperlipidemic individuals: A critical problem in design. Investig Ophthalmol Vis Sci. 2016;57(14):6339-6340. doi:10.1167/iovs.16-20099

36. Bae HW, Lee N, Lee HS, Hong S, Seong GJ, Kim CY. Systemic hypertension as a risk factor for open-angle glaucoma: A meta-analysis of population-based 
studies. PLoS One. 2014;9(9):e108226. doi:10.1371/journal.pone.0108226

37. Fraser S, Bunce C, Wormald R BE. Deprivation and late presentation of glaucoma: case control study. BMJ. 2001;322:639-643.

38. Shweikh Y, Ko F, Chan MPY, et al. Measures of socioeconomic status and self-reported glaucoma in the U.K. Biobank cohort. Eye. 2015;29(10):13601367. doi:10.1038/eye.2015.157

39. Ivers RQ, Cumming RG, Mitchell $P$, Attebo K. Visual impairment and falls in older adults: the Blue Mountains Eye Study. J Am Geriatr Soc. 1998;46(1):5864. doi:10.1111/j.1532-5415.1998.tb01014.x

40. Lamoreux EL, Chong E, Wang JJ, et al. Visual impairment, causes of vision loss, and falls: The singapore malay eye study. Investig Ophthalmol Vis Sci. 2008;49(2):528-533. doi:10.1167/iovs.07-1036

41. Höhn R, Nickels S, Schuster AK, et al. Prevalence of glaucoma in Germany: results from the Gutenberg Health Study. Graefe's Arch Clin Exp Ophthalmol. 2018;256(9):1695-1702. doi:10.1007/s00417-018-4011-z

42. Hartge P, Cahill J. Field Methods in Epidemiology. In: Rothman KJ, Greenland S, Lash TL, eds. Modern Epidemiology. 3rd ed. ; 2008:492-510.

43. Elwood JM. Commentary: On representativeness. Int J Epidemiol. 2013;42:1014-1015. doi:10.1093/ije/dyt101

44. Bourne RRA, Sukudom P, Foster PJ, et al. Prevalence of glaucoma in Thailand: a population based survey in Rom Klao District, Bangkok. $\mathrm{Br} J$ Ophthalmol. 2003;87(9):1069-1074. doi:10.1136/bjo.87.9.1069 
45. Garway-Heath DF, Rudnicka AR, Lowe T, Foster PJ, Fitzke FW, Hitchings RA. Measurement of optic disc size: Equivalence of methods to correct for ocular magnification. Br J Ophthalmol. 1998;82(6):643-649. doi:10.1136/bjo.82.6.643

46. Burr JM, Mowatt G, Hernández R, et al. The clinical effectiveness and costeffectiveness of screening for open angle glaucoma: a systematic review and economic evaluation. Health Technol Assess (Rockv). 2007;11(41):iii-iv, ix-x, 1-190. doi:04/08/02 [pii]

47. Chan MPY, Grossi CM, Khawaja AP, et al. Associations with intraocular pressure in a large cohort: Results from the UK Biobank. Ophthalmology. 2016;123(4):771-782. doi:10.1016/j.ophtha.2015.11.031

48. Jonas JB, Gusek GC, Otto G, Naumann H. Optic Disc, Cup and Neurorefinal Rim Size, Configuration and Correlations in Normal Eyes. Investig Ophthalmol Vis Sci. 1988;29(7):1151-1158.

49. Crowston JG, Hopley CR, Healey PR, Lee A, Mitchell P. The effect of optic disc diameter on vertical cup to disc ratio percentiles in a population based cohort: The Blue Mountains Eye Study. Br J Ophthalmol. 2004;88(6):766-770. doi:10.1136/bjo.2003.028548

50. Quigley HA. 21st century glaucoma care. Eye. 2019;33(2):254-260. doi:10.1038/s41433-018-0227-8

\section{Figure Legends}

Figure 1. NICOLA glaucoma prevalence study flowchart. 8,504 participants were recruited to NICOLA and had Computerised Assisted Personal Interview (CAPI). 8,265 were included in the CAPI analysis. 3,221 had vertical cup to disc ratio 
measurement in $\geq 1$ eye and comprised the denominator for the prevalence estimate. 229 particpants met the initial inclusion criteria for invitation to visual field testing and glaucoma expert review. 49 participants were excluded from invitation to visual field testing and glaucoma expert review and 180 participants were sent an invitation. 52 participants declined invitation for visual field testing and glaucoma expert review and 128 participants attended. 52 participants were diagnosed with glaucoma according to ISGEO 1 and 39 were diagnosed with glaucoma according to ISGEO 2.

Figure 2. Age-stratified (crude) prevalence of glaucoma. Bars - 95\% confidence interval 


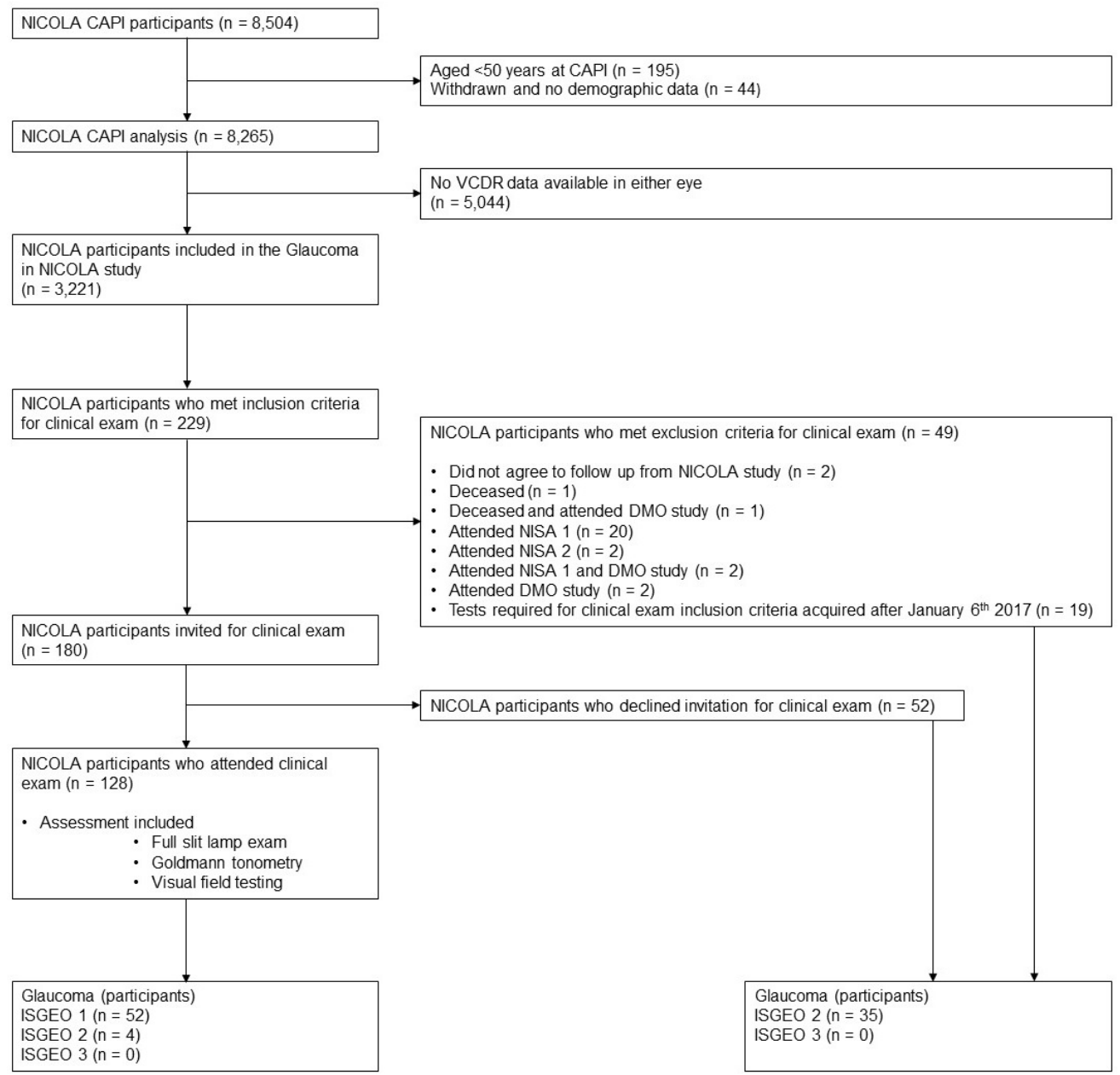

Figure 1 


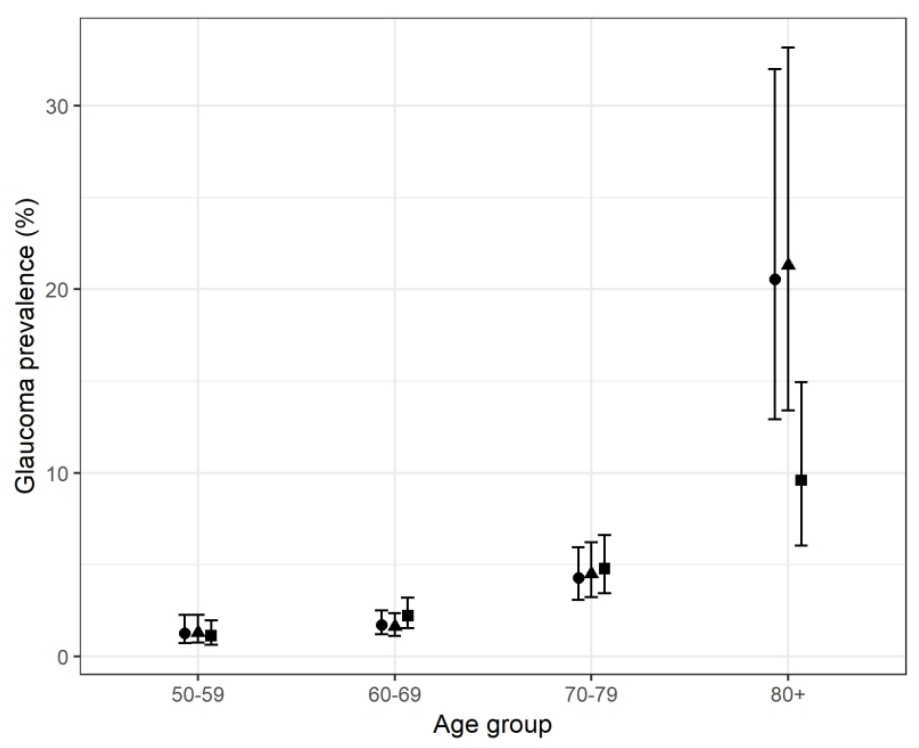

- 2011 age weighted
Estimate $\quad$ - 2017 age weighted

- Sample (crude)

Figure 2

$198 \times 116 \mathrm{~mm}(300 \times 300$ DPI $)$ 


\section{SUPPLEMENTARY MATERIAL}

\section{Supplementary Methods 1}

Ophthalmic history: Self-reported ophthalmic history was elicited from participants during the health assessment and included diagnoses of glaucoma, age related macular degeneration, cataract and diabetic retinopathy. Frequency of utilisation of optometry in the community during the previous year and history of cataract surgery in either eye were also documented.

Visual acuity (VA): VA was evaluated using mounted retro-illuminated Early Treatment Diabetic Retinopathy Study (ETDRS) LogMAR (Logarithm of the Minimum Angle of Resolution) charts for each eye in full room lighting conditions.

Refractive error: was measured in each eye as a mean of three readings using a Shin Nippon Accuref-K 900 autorefractor (Rexxam Co. Ltd., Osaka, Japan) without routine cycloplegia. Spherical equivalent (SE) was calculated as the spherical correction plus half the cylindrical power.

Ocular Response Analyser (ORA; Reichert Ophthalmic Instruments Inc, Depew, New York, USA), was used to measure the intraocular pressure (IOP) and ocular biomechanical measurements for each eye. Three measurements were taken for each eye and a maximum of five measurements were attempted for each eye. The following values were recorded at each measurement: Goldmann-correlated IOP (IOPg), corneal compensated IOP (IOPcc), corneal resistance factor (CRF), corneal hysteresis $(\mathrm{CH})$, waveform score (WFS) and the date and time of the measurement. Pupils were dilated using tropicamide $1 \%$ eye drops 
Retinal stereophotography: Digital $45^{\circ}$ colour fundus sequential stereo paired photographs centred on the optic disc were taken for each eye using a digital retinal camera (Canon CX-1, Tokyo, Japan).

Spectral-domain optical coherence tomography (SD-OCT): Spectralis Heidelberg Retina Angiograph + Optical Coherence Tomography (HRA+OCT; Heidelberg Engineering, Heidelberg, Germany) was used to obtain optic disc images which allows for circumpapillary retinal nerve fibre layer (cRNFL) thickness measurement. Each cRNFL image acquisition was manually centered on the optic disc and the following conditions were used: resolution mode: high speed; c-curve: $7.7 \mathrm{~mm}$; circle diameter: $12^{\circ}$ (fixed); size X: 768 pixels; size Z: 496 pixels; and Automatic Real Time function (ART) mode: 100 images averaged; acquisition software versions 5.10 .20 to 6.7.21.0. The cRNFL thickness was automatically segmented using Heidelberg Eye Explorer (HEYEX) software.

Spectralis HRA+OCT was used to acquire macular scans with Early Treatment Diabetic Retinopathy Study (ETDRS) and Posterior Pole Asymmetry Analysis (PPAA) maps and the following conditions were used: resolution mode: high speed; c-curve: $7.7 \mathrm{~mm}$; scan pattern size: $30.0^{\circ}$ x $25.0^{\circ}$; size X: 768 pixels; size Z: 496 pixels; ART mode: 9 images averaged; number of B-scans: 61; AQM versions 5.10.20 to 6.7.21.0. All SD-OCT scans were acquired by trained research nursing staff and assistants. Using the HEYEX software each of the 61 raster B-scans were automatically segmented into the following segment lines: Inner Limiting Membrane (ILM), Retinal Nerve Fibre Layer (RNFL), Ganglion Cell Layer (GCL), Inner Plexiform Layer (IPL), Inner Nuclear Layer (INL), Outer Plexiform Layer (OPL), External Limiting Membrane (ELM), Photoreceptors 1 (PR1), Photoreceptors 2 (PR2), Retinal Pigment Epithelium (RPE), Bruch's Membrane (BM). 
NICOLA Study image grading and quality assurance

SD-OCT cRNFL Scan Analysis: The cRNFL thickness was automatically segmented using HEYEX software. Each cRNFL scan was inspected for quality assurance purposes. Scans with minor segmentation errors were adjusted manually using the HEYEX software functions when possible. Scans judged to have significant artefact (cropped cRNFL; not centred on optic disc) or be grossly abnormal (significant vitreoretinal traction, hyporeflective intraretinal spaces, epiretinal membrane) were excluded from analysis. Fovea disc (FoDi) alignment was adjusted post acquisition if required. A proprietary measure of scan quality $(Q) \geq 15$ was considered acceptable as per the manufacturer's recommendations. Global and sectoral raw values and classifications were automatically exported.

SD-OCT ETDRS and PPAA Scan Analysis: Each of the 61 raster B-scans for each eye was reviewed by a group of trained graders and segmentation errors were manually corrected using the HEYEX software functions when possible. Macular scans which included raster B-scans with gross abnormalities (significant vitreoretinal traction, hyporeflective intraretinal spaces, epiretinal membrane) or poor segmentation which could not be corrected manually were excluded if these abnormalities were judged to render the average thickness of the overall macular scan unreliable.

Cardiovascular and anthropometric tests were performed: Two brachial blood pressure and heart rate readings were recorded at room temperature whilst seated using the M10-IT blood pressure monitor (OMRON ${ }^{\mathrm{TM}}$, Hoofddorp, The Netherlands) and the mean heart rate, mean systolic and mean diastolic blood pressure results were calculated. Body height was measured using the Seca 240 wall mounted 
measuring rod (Seca, Birmingham, UK). Weight was measured using electronic floor scales (Seca, Birmingham, UK). Waist and hip measurements were recorded using measuring tape (SECA, Birmingham, UK) when possible, excluding participants who were wheelchair bound or unable to stand. Ocular perfusion pressure $(2 / 3$ (mean arterial $B P-I O P)$ ), mean arterial pressure (diastolic BP + 1/3(systolic BP - diastolic BP)), systolic ocular perfusion pressure (systolic BP - IOP), diastolic ocular perfusion pressure (diastolic BP - IOP), waist / hip ratio and body mass index (BMI; weight $(\mathrm{kg}) /$ height $\left(\mathrm{m}^{2}\right)$ ) were calculated from available raw data.

Cognitive and psychiatric assessments: Mini-mental state examination (MMSE) and Montreal Cognitive Assessment (MCoA) were performed by the participant to assess cognitive function. Mood was assessed using the Centre for Epidemiological Studies Depression scale (CES-D).

Blood tests: Blood samples were taken for total cholesterol levels, direct low density lipoprotein (LDL), high density lipoprotein (HDL)-cholesterol, triglycerides and genotyping. 


\section{Supplementary Methods 2}

Primary grading was performed by trained graders at the Network of Ophthalmic Grading Centres UK (NetwORC UK). All graders measured the vertical cup to disc ratio (VCDR) using stereo viewers, Oculab image management software (Oculab V 3.7.98.0, Digital Healthcare, Cambridge, UK). Primary graders were also instructed to record presence of abnormalities including presence of disc haemorrhages, inferior neuroretinal rim thinning and notching. Due to the subjectivity in identifying optic disc features and estimating VCDR, a series of agreement studies and a systematic adjudication process was performed following initial grading by primary graders. Details of the agreement studies and adjudication process are described below.

Agreement analysis A: A sample of primary graders' gradings from participants that had at least 1 eye with VCDR $\geq 0.6$ and/or VCDR asymmetry $\geq 0.2$ and/or disc haemorrhages and/or neuroretinal rim thinning or notching, were re-graded (including fellow eyes) by a glaucoma expert (SPB) between $1^{\text {st }}$ September 2016 to $30^{\text {th }}$ November 2016. Inter-rater agreement analysis was performed between the glaucoma expert and the primary graders using Bland-Altman methods in 690 eyes (from 354 participants). The $95 \%$ limits of agreement (LoA) between primary graders and the glaucoma expert (SPB) were $-0.32(95 \%$ confidence interval $(95 \% \mathrm{Cl})-0.34$, $0.31)$ to $0.22(95 \% \mathrm{Cl} 0.21,0.24)$ with a statistically significant mean bias of -0.05 $(95 \% \mathrm{Cl}-0.06,-0.04)$. In $16.5 \%$ of cases the inter-rater agreement between primary graders and the glaucoma expert (SPB) showed $\geq 0.20$ difference.

Agreement analysis B: The same sample of primary graders' gradings (690 eyes from 354 participants) were also re-graded (including fellow eyes) by an experienced 
grader $(\mathrm{PMcC})$ and inter-rater agreement analysis was performed between the experienced grader and glaucoma expert (SPB). The 95\% LoA between the experienced grader and the glaucoma expert were $-0.248(95 \% \mathrm{Cl}-0.255,-0.241)$ to $0.134(95 \% \mathrm{Cl} 0.126,0.141)$ with a statistically significant mean bias of -0.057 $(95 \% \mathrm{Cl}-0.0646,-0.0500)$. In $10.0 \%$ of cases the inter-rater agreement between the experienced grader and the glaucoma expert (SPB) showed $\geq 0.20$ difference Agreement analysis C: A glaucoma expert (AAB) graded a random sample of 27 optic discs from 27 participants with VCDR $\geq 0.2$ and inter-rater agreement analysis was performed between two glaucoma experts (SPB and AAB). The 95\% LoA between the two glaucoma experts) were $-0.12(95 \% \mathrm{Cl}-0.17,-0.08)$ to $0.15(95 \% \mathrm{Cl}$ $0.10,0.19)$ with a mean bias of $0.013(95 \% \mathrm{Cl}-0.014,0.041)$ which was not statistically significant. There were no cases which showed $\geq 0.20$ difference in interrater agreement between the two glaucoma experts.

Following agreement analysis and, to account for the potential for VCDR measurements by the primary graders to disagree with the glaucoma expert (SPB) by up to 0.29 (mean bias of -0.05 to upper $95 \%$ confidence limit of the upper LOA of 0.24 equals 0.29 ), all VCDRs graded $0.5-0.6$ by the primary graders were regraded by the experienced grader (PMcC), who determined the final grading and, optic discs graded $0.41-0.5$ by primary graders were inspected for obvious signs of glaucoma by the experienced grader and only if features of glaucomatous optic neuropathy were suspected, the VCDR was re-graded by the experienced grader. The lower limit of 0.41 was selected because it is 0.29 less than 0.7 (VCDR $\geq 0.7$ was a criteria for invitation for visual field testing). 


\section{Supplementary Methods 3}

Independent variables in the multivariable association analysis with glaucoma

\begin{tabular}{|l|}
\hline Age at ISGEO classification (continuous and categorical) \\
\hline Sex \\
\hline Habitation (urban versus rural) \\
\hline Education attainment level (highest qualification attained) \\
\hline Smoking status (ex-smoker versus current smoker) and \\
\hline Smoking status (ever versus never) \\
\hline Smoking status (current versus never) \\
\hline Diabetes mellitus (self-reported history of diabetes or random glucose $\geq$ \\
11.1 mmol/L or HbA1c $\geq 6.5 \%$ or the use of antidiabetic medication) \\
\hline $\begin{array}{l}\text { Hypertension (self-reported history of high blood pressure or systolic blood } \\
\text { pressure } \geq 140 \text { mmHg or diastolic blood pressure } \geq 90 \text { mmHg or use of } \\
\text { antihypertensive medication) }\end{array}$ \\
\hline $\begin{array}{l}\text { High cholesterol (self-reported history of high cholesterol or total } \\
\text { cholesterol } \geq 6.2 \text { mmol/L or the use of lipid lowering medication) }\end{array}$ \\
\hline Cardiovascular disease (self-reported angina or heart attack) \\
\hline $\begin{array}{l}\text { Cerebrovascular disease (self-reported stroke or transient ischaemic } \\
\text { attack) }\end{array}$ \\
\hline Parkinson's disease (self-report or use of anti-Parkinsonian medication) \\
\hline Cataract surgery (self-report; right eye or left eye or both eyes) \\
\hline Body mass index (BMI) \\
\hline Height (m) \\
\hline Weight (kg) \\
\hline Waist (cm) \\
\hline Waist / hip ratio \\
\hline Spherical equivalent \\
\hline Refractive error (categorical) \\
\hline Goldmann correlated IOP (IOPg) \\
\hline Corneal compensated IOP (IOPcC) \\
\hline Corneal hysteresis (CH) \\
\hline Corneal resistance factor (CRF) \\
\hline Mean ocular perfusion pressure (MOPP) \\
\hline Systolic ocular perfusion pressure (SOPP) \\
\hline Diastolic ocular perfusion pressure (DOPP) \\
\hline Mean arterial pressure (MAP) \\
\hline Systolic blood pressure (SBP) \\
\hline Diastolic blood pressure (DBP) \\
\hline Heart rate \\
\hline $\begin{array}{l}\text { American College of Cardiology / American Heart Association (ACC/AHA) } \\
\text { hypertension stages }\end{array}$ \\
\hline Sibling history of glaucoma \\
\hline Number of falls in past 12 months \\
\hline Income domain deprivation score \\
\hline Plasma total cholesterol levels \\
\hline Plasma direct low density lipoprotein (LDL) \\
\hline Plasma high density lipoprotein (HDL)-cholesterol \\
\hline
\end{tabular}


Plasma triglycerides

Centre for Epidemiological Studies score for depression (CES-D)

National Eye Institute Visual Function Questionnaire 9 (NEI VFQ 9; composite and subscale scores)

Visits to optician in past 12 months

Overall income deprivation domain score

Mean global cRNFL thickness

Anatomical Therapeutic Chemical classifications: anti-Parkinsonian drugs (ATC N04), lipid lowering drugs (ATC C10), antihypertensive drugs (ATC C02), calcium channel blockers (ATC C08), beta blocker drugs (ATC C07) and renin-angiotensin system drugs (ATC C09) 
Supplementary Table S1. Computer assisted personal interview (CAPI) questions (Variables).

\begin{tabular}{|c|c|}
\hline CAPI Variable (Question) & CAPI Response Options \\
\hline \multirow[t]{3}{*}{ Is this dwelling located } & In Belfast city \\
\hline & $\begin{array}{l}\text { A city or town in Northern Ireland } \\
\text { other than Belfast }\end{array}$ \\
\hline & In a rural part of Northern Ireland \\
\hline \multirow{2}{*}{ Is the respondent male or female? } & Male \\
\hline & Female \\
\hline In which year were you born? & 19 \\
\hline \multirow{8}{*}{$\begin{array}{l}\text { What is the highest level of } \\
\text { education you have completed? }\end{array}$} & Some primary (not complete) \\
\hline & Primary or equivalent \\
\hline & $\begin{array}{l}\text { GCSE / O-level / Intermediate / } \\
\text { junior / group certificate or } \\
\text { equivalent }\end{array}$ \\
\hline & $\begin{array}{l}\text { A-level / Leaving certificate or } \\
\text { equivalent }\end{array}$ \\
\hline & Diploma / certificate \\
\hline & $\begin{array}{l}\text { Undergraduate Primary degree } \\
\text { Postgraduate/higher degree }\end{array}$ \\
\hline & None \\
\hline & Refused \\
\hline \multirow{7}{*}{$\begin{array}{l}\text { Have any of your brothers or sisters } \\
\text { ever suffered from any of the } \\
\text { following eye diseases? }\end{array}$} & Cataract \\
\hline & Macular Degeneration \\
\hline & Glaucoma \\
\hline & Diabetic related Eye Disease \\
\hline & None of the above \\
\hline & Do not know \\
\hline & Prefer not to answer \\
\hline \multirow{15}{*}{$\begin{array}{l}\text { Has a doctor ever told you that you } \\
\text { have any of the following } \\
\text { conditions? }\end{array}$} & High blood pressure or hypertension \\
\hline & Angina \\
\hline & $\begin{array}{l}\text { A heart attack (including myocardial } \\
\text { infarction or coronary thrombosis) }\end{array}$ \\
\hline & Congestive heart failure \\
\hline & Diabetes or high blood sugar \\
\hline & A stroke (cerebral vascular disease) \\
\hline & Mini-stroke or TIA \\
\hline & High cholesterol \\
\hline & A heart murmur \\
\hline & Atrial fibrillation \\
\hline & $\begin{array}{l}\text { Abnormal heart rhythm (not atrial } \\
\text { fibrillation) }\end{array}$ \\
\hline & Any other heart trouble (specify) \\
\hline & None of these \\
\hline & Don't Know \\
\hline & Refused \\
\hline
\end{tabular}




\begin{tabular}{|c|c|}
\hline \multirow{18}{*}{$\begin{array}{l}\text { Has a doctor ever told you that you } \\
\text { have any of the following } \\
\text { conditions? }\end{array}$} & $\begin{array}{l}\text { Chronic lung disease such as } \\
\text { chronic bronchitis or emphysema }\end{array}$ \\
\hline & Asthma \\
\hline & $\begin{array}{l}\text { Arthritis (including osteoarthritis, or } \\
\text { rheumatism) }\end{array}$ \\
\hline & $\begin{array}{l}\text { Osteoporosis, sometimes called thin } \\
\text { or brittle bones }\end{array}$ \\
\hline & $\begin{array}{l}\text { Cancer or a malignant tumour } \\
\text { (including leukaemia or lymphoma } \\
\text { but excluding minor skin cancers) }\end{array}$ \\
\hline & Parkinson's disease \\
\hline & $\begin{array}{l}\text { Any emotional, nervous or } \\
\text { psychiatric problems such as } \\
\text { depression or anxiety }\end{array}$ \\
\hline & Alcohol abuse \\
\hline & Substance abuse \\
\hline & Alzheimer's disease \\
\hline & Dementia \\
\hline & Serious memory impairment \\
\hline & Stomach ulcers \\
\hline & $\begin{array}{l}\text { Varicose Ulcers (an ulcer due to } \\
\text { varicose veins) }\end{array}$ \\
\hline & Cirrhosis, or serious liver damage \\
\hline & None of these \\
\hline & Don't Know \\
\hline & Refused \\
\hline $\begin{array}{l}\text { How many times have you fallen in } \\
\text { the last year? }\end{array}$ & - \\
\hline \multirow{4}{*}{$\begin{array}{l}\text { Have you ever smoked cigarettes, } \\
\text { cigars, cigarillos or a pipe daily for a } \\
\text { period of at least one year? }\end{array}$} & 8 \\
\hline & 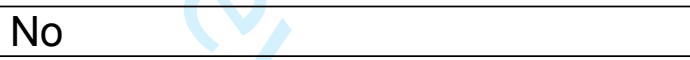 \\
\hline & Don't know \\
\hline & Refused \\
\hline \multirow[t]{4}{*}{ Do you smoke at the present time? } & Yes (in the past 3 months) \\
\hline & No, I have stopped \\
\hline & Don't know \\
\hline & Refused \\
\hline Medication history & $\begin{array}{l}\text { Anatomical Therapeutic Chemical } \\
\text { (ATC) classification }\end{array}$ \\
\hline $\begin{array}{l}\text { Overall income deprivation domain } \\
\text { score }\end{array}$ & $\begin{array}{l}\text { Proportion of population } \\
\text { experiencing income deprivation in } \\
\text { an area (super output area) }\end{array}$ \\
\hline House & \\
\hline
\end{tabular}


Supplementary Table S2. NEI VFQ 9 Questions

\begin{tabular}{|c|c|}
\hline Q1 & At the present time would you say your eyesight is \\
\hline Q2 & How much time do you worry about your eyesight? \\
\hline Q3 & How much difficulty do you have reading ordinary print newspaper? \\
\hline Q4 & $\begin{array}{l}\text { How much difficulty do you have doing work or hobbies that require } \\
\text { you to see well up close such as cooking, sewing, fixing things } \\
\text { around the house or using hand tools? }\end{array}$ \\
\hline Q5 & $\begin{array}{l}\text { Because of your eyesight how much difficulty do you have going } \\
\text { down steps, stairs or curbs in dim light or at night? }\end{array}$ \\
\hline Q6 & $\begin{array}{l}\text { How much difficulty do you have driving during daytime in familiar } \\
\text { places? }\end{array}$ \\
\hline Q7 & $\begin{array}{l}\text { Are you limited in how long you can walk or other activities such as } \\
\text { housework, childcare, school or community activities because of } \\
\text { your eyesight? }\end{array}$ \\
\hline Q8 & $\begin{array}{l}\text { Because of your eyesight how much difficulty do you have noticing } \\
\text { objects off to the side while you are walking along? }\end{array}$ \\
\hline Q9 & $\begin{array}{l}\text { Because of your eyesight how much difficulty do you have finding } \\
\text { something off a crowded shelf? }\end{array}$ \\
\hline
\end{tabular}

The scale for question 1 was, 1) excellent, 2) good, 3) fair, 4) poor, and 5) completely blind. The scales for questions 2 to 9 were, 1) no difficulty, 2) a little difficulty, 3) moderate difficulty, 4) extreme difficulty, and 5) stopped doing because of eyesight. The option 'stopped doing for other reasons' was also available for several subscales. 
Supplementary Table S3. International Society Geographical and Epidemiological Ophthalmology (ISGEO) criteria - Diagnosis of glaucoma and glaucoma related findings in cross sectional prevalence surveys

\begin{tabular}{|c|c|}
\hline $\begin{array}{l}\text { ISGEO category } 1 \\
\text { diagnosis (structural } \\
\text { and functional } \\
\text { evidence) }\end{array}$ & $\begin{array}{l}\text { Eyes with a cup to disc ratio (CDR) or CDR asymmetry } \geq 97.5 \text { th percentile for the normal population } \\
(\geq 0.7) \text {, or a neuroretinal rim width reduced to } \leq 0.1 \text { CDR (between } 11 \text { to } 1 \text { o'clock or } 5 \text { to } 7 \text { o'clock) that } \\
\text { also showed a definite visual field defect consistent with glaucoma (see footnote). }\end{array}$ \\
\hline $\begin{array}{l}\text { ISGEO category } 2 \\
\text { diagnosis (advanced } \\
\text { structural damage } \\
\text { with unproved field } \\
\text { loss) }\end{array}$ & $\begin{array}{l}\text { If the subject could not satisfactorily complete visual field testing but had a CDR or CDR asymmetry } \\
\geq 99.5 \text { th percentile for the normal population }(\geq 0.8) \text {, glaucoma was diagnosed solely on the structural } \\
\text { evidence. }\end{array}$ \\
\hline $\begin{array}{l}\text { ISGEO category } 3 \\
\text { diagnosis (Optic disc } \\
\text { not seen. Field test } \\
\text { impossible) }\end{array}$ & $\begin{array}{l}\text { If it is not possible to examine the optic disc, glaucoma is diagnosed if: } \\
\text { (A) The visual acuity }<3 / 60 \text { and the IOP }>99.5 \text { th percentile, or } \\
\text { (B) The visual acuity }<3 / 60 \text { and the eye shows evidence of glaucoma filtering surgery, or medical } \\
\text { records were available confirming glaucomatous visual morbidity. }\end{array}$ \\
\hline Disc suspects & Those who met ISGEO 1 criteria for disc parameters but were not found to have visual field defects. \\
\hline Ocular hypertension & Those with an IOP $>21 \mathrm{mmHg}$. \\
\hline \multicolumn{2}{|c|}{$\begin{array}{l}\text { Footnote: In diagnosing category } 1 \text { or } 2 \text { glaucoma, there should be no alternative explanation for CDR findings (dysplastic disc } \\
\text { or marked anisometropia) or the visual field defect (retinal vascular disease, macular degeneration, or cerebrovascular } \\
\text { disease). Gold standard of glaucomatous visual field loss - glaucoma hemifield test graded "outside normal limits", a cluster of } \\
\text { three contiguous points at the } 5 \% \text { level on the pattern deviation plot, using the threshold test strategy with the } 24-2 \text { test pattern } \\
\text { of the Zeiss-Humphrey field analyser } 2 \text { (not the only acceptable tool for field analysis). }\end{array}$} \\
\hline
\end{tabular}


Supplementary Table S4. Number of missing measurements for vertical cup to disc ratio, Goldman correlated intraocular pressure (IOPg) and circumpapillary retinal nerve fibre layer (cRNFL) among the 3,221 included participants

\begin{tabular}{|l|l|l|l|}
\hline Parameter Type & $\begin{array}{l}\text { Number of right eye missing } \\
\text { measurements (\%) }\end{array}$ & $\begin{array}{l}\text { Number of left eye missing } \\
\text { measurements (\%) }\end{array}$ & $\begin{array}{l}\text { Number of participants with measurements } \\
\text { missing bilaterally (\%) }\end{array}$ \\
\hline VCDR & $94(2.9 \%)$ & $135(4.2 \%)$ & n/a \\
\hline IOPg & $335(10.4 \%)$ & $354(11.1 \%)$ & $306(9.5 \%)$ \\
\hline cRNFL & $458(14.2 \%)$ & $523(16.2 \%)$ & $209(6.5 \%)$ \\
\hline
\end{tabular}


Supplementary Table S5. Baseline characteristics of NICOLA participants eligible for the GwNICOLA study and comparisons between GwNICOLA attendees and nonattendees.

\begin{tabular}{|c|c|c|c|c|}
\hline & $\begin{array}{l}\text { GwNICOLA } \\
\text { cohort } \\
(n=229)\end{array}$ & $\begin{array}{l}\text { GwNICOLA } \\
\text { attendees } \\
(n=128)\end{array}$ & $\begin{array}{l}\text { GwNICOLA } \\
\text { non- } \\
\text { attendees } \\
(\mathrm{n}=101)\end{array}$ & $P$ value \\
\hline $\begin{array}{l}\text { Age at NICOLA } \\
\text { health assessment } \\
(n=229)\end{array}$ & $67.70(9.14)$ & $66.62(8.66)$ & $69.07(9.58)$ & 0.044 \\
\hline $\begin{array}{l}\text { Sex } \\
(n=229)\end{array}$ & & & & 0.160 * \\
\hline Male (\%) & $115(50.2)$ & $59(46.1)$ & $56(55.4)$ & \\
\hline Female (\%) & $114(49.8)$ & 69 (53.9) & $45(44.6)$ & \\
\hline $\begin{array}{l}\text { Habitation } \\
(n=183)\end{array}$ & 8 & & & 0.551 * \\
\hline $\begin{array}{l}\text { Urban / city / } \\
\text { townland (\%) }\end{array}$ & $139(76.0)$ & 75 (74.3) & $64(78.0)$ & \\
\hline Rural (\%) & $44(24.0)$ & $26(25.7)$ & $18(22.0)$ & \\
\hline $\begin{array}{l}\text { Median income } \\
\text { domain score (IQR) } \\
(\mathrm{n}=228)\end{array}$ & $\begin{array}{l}0.1900 \\
(0.1125 \\
0.2800)\end{array}$ & $\begin{array}{l}0.1950 \\
(0.1100 \\
0.2800)\end{array}$ & $\begin{array}{l}0.1900 \\
(0.1300 \\
0.2800)\end{array}$ & $0.832 \ddagger$ \\
\hline $\begin{array}{l}\text { Median house value } \\
(\text { IQR) } \\
(\mathrm{n}=228)\end{array}$ & $\begin{array}{l}135000 \\
(95000, \\
190000)\end{array}$ & $\begin{array}{l}136000 \\
(100000, \\
195000)\end{array}$ & $\begin{array}{l}132000 \\
(87750, \\
190000)\end{array}$ & $0.375 \ddagger$ \\
\hline $\begin{array}{l}\text { Median NEI VFQ } \\
\text { score (IQR) } \\
(\mathrm{n}=224)\end{array}$ & $\begin{array}{l}12.00 \\
(11.00 \\
15.00)\end{array}$ & $\begin{array}{l}12.50 \\
(11.00 \\
15.00) \\
\end{array}$ & $\begin{array}{l}12.00 \\
(10.00 \\
15.00)\end{array}$ & $0.301 \ddagger$ \\
\hline \multicolumn{5}{|l|}{ Mean VCDR (SD) } \\
\hline $\begin{array}{l}\text { Right eye } \\
(\mathrm{n}=219)\end{array}$ & $0.60(0.17)$ & $0.58(0.16)$ & $0.63(0.17)$ & 0.012 \\
\hline $\begin{array}{l}\text { Left eye } \\
(n=218)\end{array}$ & $0.60(0.18)$ & $0.60(0.18)$ & $0.62(0.19)$ & 0.245 \\
\hline Mean IOPg (SD) & & & +2 & \\
\hline $\begin{array}{l}\text { Right eye } \\
(n=212)\end{array}$ & $18.37(5.43)$ & $18.26(5.33)$ & $18.50(5.59)$ & 0.752 \\
\hline $\begin{array}{l}\text { Left eye } \\
(\mathrm{n}=210)\end{array}$ & $18.46(5.70)$ & $18.72(5.60)$ & $18.10(5.84)$ & 0.432 \\
\hline \multicolumn{5}{|l|}{$\begin{array}{l}\text { Mean Spherical } \\
\text { equivalent (SD) }\end{array}$} \\
\hline $\begin{array}{l}\text { Right eye } \\
(\mathrm{n}=209)\end{array}$ & $0.33(2.65)$ & $0.30(2.81)$ & $0.35(2.45)$ & 0.892 \\
\hline $\begin{array}{l}\text { Left eye } \\
(n=211)\end{array}$ & $0.31(2.59)$ & $0.39(2.59)$ & $0.21(2.59)$ & 0.605 \\
\hline
\end{tabular}


Supplementary Table S6. ISGEO classifications by eye

\begin{tabular}{|l|l|l|l|}
\hline & ISGEO 1 & ISGEO 2 & ISGEO 3 \\
\hline Right eyes & 36 & $25^{\mathrm{b}}$ & 0 \\
\hline Left eyes & 40 & $29^{\mathrm{b}}$ & 0 \\
\hline a Participants & 52 & $39^{\mathrm{c}}$ & 0 \\
\hline
\end{tabular}

a - Participants counted once using highest level of evidence available: ISGEO $1>$ ISGEO 2 > ISGEO $3 ; b-5$ right eyes and 5 left eyes from NICOLA participants who attended the clinical exam were diagnosed with glaucoma according to ISGEO category 2; C - ISGEO category 2 definition was the highest quality evidence of glaucoma in 4 participants who attended the clinical exam. 
Supplementary Table S7. Reasons for unreliable visual field tests (or abnormal visual fields not due to glaucoma) are outlined

\begin{tabular}{|l|l|l|}
\hline & Right eye & Left eye \\
\hline Homonomous hemianopia & 3 & 3 \\
\hline Bitemporal hemianopia & 1 & 1 \\
\hline Reliability indices & 1 & 1 \\
\hline Cataract with logMAR $\geq 0.3$ & 2 & 2 \\
\hline $\begin{array}{l}\text { Media opacity or refractive error or } \\
\text { amblyopia with logMAR } \geq 0.3\end{array}$ & 4 & 4 \\
\hline Retinal vein occlusion & 2 & 0 \\
\hline Retinal arterial occlusion & 0 & 1 \\
\hline Macular hole surgery & 1 & 1 \\
\hline Retinal detachment surgery & 2 & 1 \\
\hline
\end{tabular}

